\title{
A new general relativistic magnetohydrodynamics code for dynamical spacetimes
}

\author{
P. Cerdá-Durán ${ }^{1}$, J. A. Font ${ }^{2}$, L. Antón ${ }^{2}$, and E. Müller ${ }^{1}$ \\ 1 Max-Planck-Institut für Astrophysik, Karl-Schwarzschild-Str. 1, 85741 Garching, Germany \\ e-mail: cerda@mpa-garching.mpg.de \\ 2 Departamento de Astronomía y Astrofísica, Universidad de Valencia, 46100 Burjassot (Valencia), Spain
}

Received 29 April 2008 / Accepted 18 September 2008

ABSTRACT

\begin{abstract}
We present a new numerical code that solves the general relativistic magneto-hydrodynamical (GRMHD) equations coupled to the Einstein equations for the evolution of a dynamical spacetime within a conformally-flat approximation. This code has been developed with the main objective of studying astrophysical scenarios in which both, high magnetic fields and strong gravitational fields appear, such as the magneto-rotational collapse of stellar cores, the collapsar model of GRBs, and the evolution of neutron stars. The code is based on an existing and thoroughly tested purely hydrodynamical code and on its extension to accommodate weakly magnetized fluids (passive magnetic-field approximation). These codes have been applied in the past to simulate the aforementioned scenarios with increasing levels of sophistication in the input physics. The numerical code we present here is based on high-resolution shockcapturing schemes to solve the GRMHD equations, which are cast in first-order, flux-conservative hyperbolic form, together with the flux constraint transport method to ensure the solenoidal condition of the magnetic field. Since the astrophysical applications envisaged do not deviate significantly from spherical symmetry, the conformal flatness condition approximation is used for the formulation of the Einstein equations; this has repeatedly shown to yield very good agreement with full general relativistic simulations of corecollapse supernovae and the evolution of isolated neutron stars. In addition, the code can handle several equations of state, from simple analytical expressions to microphysical tabulated ones. In this paper we present stringent tests of our new GRMHD numerical code, which show its ability to handle all aspects appearing in the astrophysical scenarios for which the code is intended, namely relativistic shocks, highly magnetized fluids, and equilibrium configurations of magnetized neutron stars. As an application, magnetorotational core-collapse simulations of a realistic progenitor are presented and the results compared with our previous findings in the passive magnetic-field approximation.
\end{abstract}

Key words. gravitation - hydrodynamics - magnetohydrodynamics (MHD) - methods: numerical - relativity stars: supernovae: general

\section{Introduction}

The collapse of rotating stellar cores and the merging of compact binaries (either neutron star-neutron star or neutron star-black hole binaries) are two of the most important astrophysical scenarios involving compact objects, whose modeling requires the study of the dynamical evolution of a magnetized fluid in general relativity. In the case of the rotational collapse of massive stellar cores, the magnetic field is thought to grow through the extraction of energy from the differential rotation generated during collapse (Meier et al. 1976). This idea is supported by the observational fact that some neutron stars (magnetars) possess extremely large magnetic fields $\left(10^{14}-10^{15}\right.$ Gauss), as inferred from studies of anomalous X-ray pulsars and soft gamma-ray repeaters (Kouveliotou et al. 1998). Furthermore, the class of soft-long gamma-ray bursts (GRB) are probably the result of jets formed in a rotational core-collapse event leading to a black hole, according to the collapsar scenario (Woosley et al. 1993). In this case, the magnetic field most likely plays a crucial role in the formation and collimation of the jet. This scenario is supported by the existing correlation of some long GRB with corecollapse supernova events (see Kelly et al. 2007, and references therein). In the case of the mergers of two neutron stars, believed to be the standard mechanism to account for hard-short GRBs, it was suggested (Price \& Rosswog 2006) that the strong shear between the two neutron stars could generate strong magnetic fields, too.

A considerable effort has been made to develop special relativistic magneto-hydrodynamics (SRMHD) codes (see e.g. Martí \& Müller 2002; Ibáñez 2006, and references therein). Most works have considered the case of ideal MHD where the fluid is assumed to be a perfect conductor. In this case, the resulting system of equations is simplified significantly, and can be solved by numerical codes designed specifically for hyperbolic systems. These codes include the use of Godunov-type schemes (Komissarov 1999), numerical techniques to keep the magnetic field divergence-free (see Tóth 2000, and references therein), and efficient recovery schemes to derive primitive quantities from the conserved ones (see Noble et al. 2006, and references therein). There is also a major activity in the development of codes capable of simulating magnetized astrophysical flows in general relativity. These codes integrate the ideal GRMHD equations for fixed background spacetimes using high-order conservative schemes based on either approximate or full wave-decomposition Riemann solvers (Gammie et al. 2003; Komissarov 2005; Anninos et al. 2005; Antón et al. 2006; Del Zanna et al. 2007; Tchekhovskoy et al. 2007). Resistive MHD flows were considered by Komissarov (2007), and nonconservative GRMHD schemes and schemes relying on artificial viscosity were used by De Villiers \& Hawley (2003) and 
Anninos et al. (2005). Most codes have been applied to study disk accretion onto black holes and jet formation, but since the self-gravity of the fluid was not taken into account, these codes cannot simulate consistently the formation of the black hole and the evolution of the surrounding disk or torus.

Only very recently, GRMHD codes are able to follow the evolution of a dynamical spacetime. The codes of Duez et al. (2005), Shibata \& Sekiguchi (2005), and Giacomazzo \& Rezzolla (2007) are based on the BSSN formulation of the Einstein equations for the spacetime (Nakamura et al. 1987; Shibata \& Nakamura 1995; Baumgarte \& Shapiro 1999), high resolution shock-capturing schemes for the GRMHD equations involving approximate Riemann solvers such as HLL or highorder central schemes, and on the constraint transport scheme for the magnetic field. In the code of Anderson et al. (2008), the Einstein equations are cast in first-order symmetric hyperbolic form, and are solved using the generalized harmonic decomposition. While the code relies on the same type of GR hydrodynamics solvers as previously developed codes, it guarantees a divergence-free magnetic field by means of either projection methods or hyperbolic divergence cleaning. Both methods are easier to implement for the non-structured AMR grids employed in this code than the constrained transport method. All four codes rely on Cartesian coordinates for three dimensional simulations. The codes of Duez et al. (2005) and Shibata $\&$ Sekiguchi (2005) also provide the possibility to impose axisymmetry by means of the cartoon method for the spacetime evolution and the use of cylindrical coordinates for the GRMHD equations. The equations of state (EOS) implemented in these codes consist of simple analytic expressions: polytropic EOS, ideal gas or hybrid EOS (see Sect. 2.3). One of the codes was extended to handle a tabulated microphysical EOS (Shibata et al. 2007).

We present a new axisymmetric numerical code, capable of handling ideal MHD flows in dynamical spacetimes in general relativity, and designed particularly to investigate gravitational core collapse. We use similar numerical schemes as in most of the other existing GRMHD codes (HRSC schemes and constraint transport), but we follow a simpler approach for the spacetime evolution.

The new code is based on the hydrodynamics code described in Dimmelmeier et al. (2002a,b), and on its extensions discussed in Cerdá-Durán et al. (2005), Cerdá-Durán \& Font (2007), and Cerdá-Durán et al. (2007). The Maxwell equations are already incorporated in the codes of Cerdá-Durán \& Font (2007) and Cerdá-Durán et al. (2007), but only in the passive magnetic-field approximation, i.e. the contribution of the magnetic field to the energy-momentum tensor is neglected, and therefore has no impact on the dynamics. In the new code, we relax this assumption and incorporate magnetic field effects on the spacetime dynamics and the self-gravity of the fluid following the approach laid out in Antón et al. (2006). The Einstein equations are formulated using the conformal flatness condition (CFC hereafter). This approximate treatment of the metric equations was first introduced by Isenberg (2008) and Wilson et al. (1996), and was used to study rotational core collapse (Dimmelmeier et al. 2002a,b), and binary neutron stars (Oechslin et al. 2007). Simulations with a second post-Newtonian extension of the CFC metric (named $\mathrm{CFC}+$ ) showed small quantitative differences in the dynamics and the gravitational waveforms $(<1 \%)$ compared to the CFC metric, both for rotational core collapse and for simulations of the evolution of single neutron stars (Cerdá-Durán et al. 2005). Direct comparisons of the CFC approach with full general relativistic simulations were reported by Shibata \& Sekiguchi (2004) and Ott et al. (2007a,b), who found that the differences in the collapse dynamics and the waveforms are minute demonstrating the suitability of CFC for performing accurate core collapse simulations.

The CFC approach has some advantages compared with the BSSN formulation: (i) the Hamiltonian and momentum constraints of the Einstein equations are automatically satisfied; and (ii) the time step is less restrictive since it is determined by the largest fluid eigenvalue, while in hyperbolic formulations (such as BSSN) the largest eigenvalue is the speed of light. Consequently, the time steps are typically ten times larger than those admitted in BSSN for the same grid for the evolution of neutron stars, and even larger during core collapse. However, there are also some disadvantages of the CFC approach. It neglects the gravitational wave content of the spacetime, i.e. when the gravitational wave back reaction is important (e.g. in neutron-star mergers) the dynamics cannot be modeled accurately (e.g. compare the simulations of Oechslin et al. 2007 and Shibata \& Taniguchi 2006). Furthermore, to compute the gravitational waveforms one needs to resort to the Einstein quadrupole formula, and, since the CFC metric equations are elliptic, the parallelization of the code for a large number of processors is more difficult than in hyperbolic formulations such as BSSN, but still possible.

Our code uses spherical polar coordinates and (presently) assumes axisymmetry. The most important advantage of these coordinates with respect to Cartesian or cylindrical coordinates adopted by other numerical codes, is that they are more readily adapted to the astrophysical scenarios that we wish to study. Furthermore, it allows us to easily and properly cover the lengthscales of core collapse ranging from the radius of the initial iron core $(\sim 1000 \mathrm{~km})$ down to the radius of the neutron star $(\sim 10 \mathrm{~km})$ by means of a logarithmically spaced radial grid. A disadvantage of our coordinate system concerns its possible extension to 3D because of the coordinate singularities at the center and at the axis. Moreover, as the azimuthal grid spacing decreases quadratically towards the axis with increasing grid resolution (for an equally-spaced angular grid), the Courant condition for the time step can be rather restrictive in 3D simulations (a possible solution to this issue can be found e.g. in Zink et al. 2008).

The code can handle various equations of state ranging from simple analytical expressions (polytropes, ideal gas and hybrid EOS) to tabulated microphysical EOS. General relativistic hydrodynamic core-collapse simulations using the tabulated EOS were performed by Ott et al. (2007a) and Dimmelmeier et al. (2007), and magneto-hydrodynamic simulations by Cerdá-Durán et al. (2007) using the passive magneticfield approximation. These three studies also included a simplified treatment of neutrino transport.

The rest of the paper is organized as follows. Section 2 presents a brief overview of the theoretical framework we use, namely the CFC equations and the GRMHD equations in the $3+1$ formalism. Our numerical approach is discussed in Sect. 3 . Tests of the numerical code are presented in Sect. 4 including a magneto-rotational core collapse simulation, and the conclusions are given in Sect. 5. Throughout the paper, we use a spacelike metric signature $(-,+,+,+)$, and units where $c=G=1$. We absorb the factor $1 / \sqrt{4 \pi}$ appearing in the MHD equations in the definition of the magnetic field $B^{i}$, i.e. the units of the magnetic field are $\sqrt{4 \pi}$ Gauss. Greek indices run from 0 to 3, Latin indices from 1 to 3, and we adopt the standard Einstein summation convention. 


\section{Physical approach}

We adopt the $3+1$ formalism of general relativity (Lichnerowicz 1944) to foliate the spacetime into spacelike hypersurfaces. In this approach, the line element reads

$\mathrm{d} s^{2}=-\alpha^{2} \mathrm{~d} t^{2}+\gamma_{i j}\left(\mathrm{~d} x^{i}+\beta^{i} \mathrm{~d} t\right)\left(\mathrm{d} x^{j}+\beta^{j} \mathrm{~d} t\right)$,

where $\alpha$ is the lapse function, $\beta^{i}$ is the shift vector, and $\gamma_{i j}$ is the spatial three-metric induced in each hypersurface. Using the projection operator $\perp_{v}^{\mu}$ and the unit four-vector $n^{\mu}$ normal to each hypersurface, it is possible to build the quantities

$$
\begin{aligned}
E & \equiv n^{\mu} n^{\nu} T_{\mu v}=\alpha^{2} T^{00}, \\
S_{i} & \equiv-\perp_{i}^{\mu} n^{\nu} T_{\mu \nu}=-\frac{1}{\alpha}\left(T_{0 i}-T_{i j} \beta^{j}\right), \\
S_{i j} & \equiv \perp_{i}^{\mu} \perp_{j}^{v} T_{\mu \nu}=T_{i j},
\end{aligned}
$$

which represent the total energy, the momenta, and the spatial components of the energy-momentum tensor $T_{\mu \nu}$, respectively.

To solve the gravitational field equations we choose the ADM gauge in which the three-metric can be decomposed as $\gamma_{i j}=\phi^{4} \hat{\gamma}_{i j}+h_{i j}^{\mathrm{TT}}$, where $\phi$ is the conformal factor, $\hat{\gamma}_{i j}$ is the flat three-metric, and $h_{i j}^{\mathrm{TT}}$ is the transverse and traceless part of the three-metric. We note that this gauge choice implies the maximal slicing condition where the trace $K$ of the extrinsic curvature tensor $K_{i j}$ vanishes.

\subsection{The CFC approximation}

In our work, Einstein's field equations are formulated and solved using the conformally flat condition (CFC hereafter), introduced by Isenberg (2008) and first used in a dynamical context by Wilson et al. (1996). In this approximation, the three-metric in the ADM gauge is assumed to be conformally flat, $\gamma_{i j}=\phi^{4} \hat{\gamma}_{i j}$. We note that this approximation can also be realized for other gauge choices such as the quasi-isotropic gauge or the Dirac gauge, both supplemented by the maximal slicing condition. Under the CFC assumption, the gravitational field equations can be written as a system of five nonlinear elliptic equations,

$$
\begin{aligned}
\hat{\Delta} \phi & =-2 \pi \phi^{5}\left(E+\frac{K_{i j} K^{i j}}{16 \pi}\right) \\
\hat{\Delta}(\alpha \phi) & =2 \pi \alpha \phi^{5}\left(E+2 S+\frac{7 K_{i j} K^{i j}}{16 \pi}\right) \\
\hat{\Delta} \beta^{i} & =16 \pi \alpha \phi^{4} S^{i}+2 \phi^{10} K^{i j} \hat{\nabla}_{j}\left(\frac{\alpha}{\phi^{6}}\right)-\frac{1}{3} \hat{\nabla}^{i} \hat{\nabla}_{k} \beta^{k},
\end{aligned}
$$

where $\hat{\Delta}$ and $\hat{\nabla}$ are the Laplace and nabla operators associated with the flat three-metric, and $S \equiv \gamma^{i j} S_{i j}$.

\subsection{General relativistic magnetohydrodynamics}

The energy-momentum tensor of a magnetized perfect fluid can be written as the sum of the fluid part and the electromagnetic field part. In the so-called ideal MHD limit (where the fluid is a perfect conductor of infinite conductivity), the latter can be expressed solely in terms of the magnetic field $b^{\mu}$ measured by a comoving observer. In this case, the total energy-momentum tensor is given by

$T^{\mu v}=\left(\rho h+b^{2}\right) u^{\mu} u^{v}+\left(P+\frac{b^{2}}{2}\right) g^{\mu \nu}-b^{\mu} b^{v}$, where $\rho$ is the rest-mass density, $h=1+\epsilon+P / \rho$ the relativistic enthalpy, $\epsilon$ the specific internal energy, $P$ the fluid pressure, $u^{\mu}$ the four-velocity of the fluid, and $b^{2}=b^{\mu} b_{\mu}$. We define the magnetic pressure $P_{\text {mag }}=b^{2} / 2$ and the specific magnetic energy $\epsilon_{\mathrm{mag}}=b^{2} /(2 \rho)$, whose effect on the dynamics is similar to that of the fluid pressure and the specific internal energy of the fluid, respectively.

For an Eulerian observer, $u^{\mu}=n^{\mu}$, and in the ideal MHD limit, the temporal component of the electric field vanishes, $E^{\mu}=\left(0,-\varepsilon_{i j k} v^{j} B^{k}\right)$, where $\varepsilon_{i j k}$ is the permutation tensor and $B^{k}$ is the magnetic field. In this case, Maxwell's equations reduce to the divergence-free condition and the induction equation for the magnetic field,

$\hat{\nabla}_{i} B^{* i}=0, \quad \frac{\partial B^{* i}}{\partial t}=\hat{\nabla}_{j}\left(v^{* i} B^{* j}-v^{* j} B^{* i}\right)$,

with $B^{* i} \equiv \sqrt{\bar{\gamma}} B^{i}$ and $v^{* i} \equiv \alpha v^{i}-\beta^{i}$, where $v^{i}$ is the fluid threevelocity as measured by the Eulerian observer. The ratio of the determinants of the three-metric and the flat three-metric is given by $\bar{\gamma}=\gamma / \hat{\gamma}$.

The evolution of a magnetized fluid is determined by the conservation law of the energy-momentum, $\nabla_{\mu} T^{\mu \nu}=0$, and by the continuity equation, $\nabla_{\mu} J^{\mu}=0$, for the rest-mass current $J^{\mu}=\rho u^{\mu}$. Following the procedure described by Antón et al. (2006), the conserved quantities are chosen in a way similar to the purely hydrodynamic case presented by Banyuls et al. (1997):

$$
\begin{aligned}
D & =\rho W \\
S_{i} & =\left(\rho h+b^{2}\right) W^{2} v_{i}-\alpha b_{i} b^{0} \\
\tau & =\left(\rho h+b^{2}\right) W^{2}-\left(P+\frac{b^{2}}{2}\right)-\alpha^{2}\left(b^{0}\right)^{2}-D
\end{aligned}
$$

where $W=\alpha u^{0}$ is the Lorentz factor. With this choice, the system of conservation equations for the fluid, and the induction equation for the magnetic field can be cast as a first-order, fluxconservative, hyperbolic system,

$$
\frac{1}{\sqrt{-g}}\left[\frac{\partial \sqrt{\gamma} \boldsymbol{U}}{\partial t}+\frac{\partial \sqrt{-g} \boldsymbol{F}^{i}}{\partial x^{i}}\right]=\boldsymbol{S},
$$

with the state vector, the flux vector, and the source vector given by

$$
\begin{aligned}
\boldsymbol{U}= & {\left[D, S_{j}, \tau, B^{k}\right], } \\
\boldsymbol{F}^{i}= & {\left[D \hat{v}^{i}, S_{j} \hat{v}^{i}+\delta_{j}^{i}\left(P+\frac{b^{2}}{2}\right)-\frac{b_{j} B^{i}}{W},\right.} \\
& \left.\tau \hat{v}^{i}+\left(P+\frac{b^{2}}{2}\right) v^{i}-\alpha \frac{b^{0} B^{i}}{W}, \hat{v}^{i} B^{k}-\hat{v}^{k} B^{i}\right], \\
\boldsymbol{S}= & {\left[0, \frac{1}{2} T^{\mu \nu} \frac{\partial g_{\mu \nu}}{\partial x^{j}}, \alpha\left(T^{\mu 0} \frac{\partial \ln \alpha}{\partial x^{\mu}}-T^{\mu v} \Gamma_{\mu \nu}^{0}\right), 0^{k}\right], }
\end{aligned}
$$

where $\delta_{j}^{i}$ is the Kronecker delta, and $\Gamma_{\mu \lambda}^{\mu}$ are the Christoffel symbols associated with the four-metric. We note that the above definitions contain components of the magnetic field measured by both a comoving observer and an Eulerian observer. The two are related by

$b^{0}=\frac{W B^{i} v_{i}}{\alpha}, \quad b^{i}=\frac{B^{i}+\alpha b^{0} u^{i}}{W}$. 
The hyperbolic structure of Eq. (13) and the associated spectral decomposition (into eigenvalues and eigenvectors) of the fluxvector Jacobians are given in Antón et al. (2006). This information is required to numerically solve the system of equations using the class of high-resolution shock-capturing schemes that we have implemented in our code.

\subsection{Equation of state}

The new numerical code can handle a variety of equations of state including a polytropic EOS, an ideal gas EOS, a hybrid EOS, and a tabulated microphysical EOS.

\subsubsection{Hybrid EOS}

The hybrid EOS (Janka et al. 1993) is a simplified analytical equation of state used in core collapse simulations. The pressure is given by a polytropic part, $P_{\mathrm{p}}=K \rho^{\gamma}$, with $K=4.897 \times 10^{14}$ (in cgs units), plus a thermal part, $P_{\mathrm{th}}=\rho \epsilon_{\mathrm{th}}\left(\gamma_{\mathrm{th}}-1\right)$, where the specific thermal energy, $\epsilon_{\mathrm{th}}=\epsilon-\epsilon_{\mathrm{p}}$, and $\gamma_{\mathrm{th}}=1.5$. The thermal contribution takes into account the increase of the thermal energy due to shock heating. When $\rho$ exceeds the nuclear saturation density, $\rho_{\text {nuc }}=2.0 \times 10^{14} \mathrm{~g} \mathrm{~cm}^{-3}$, the value of $\gamma$ is raised to $\gamma_{2}=2.5$, and $K$ is adjusted accordingly to guarantee the continuity of $P$ and $\epsilon$. Due to this stiffening of the EOS the core undergoes a so-called pressure-supported bounce. More details about the hybrid EOS can be found, e.g. in Dimmelmeier et al. (2002a).

\subsubsection{Microphysical EOS}

We further employ the tabulated non-zero temperature nuclear EOS by Shen et al. (1998) in the variant of Marek et al. (2005), which includes baryonic, electronic, and photonic pressure components. It specifies the fluid pressure $P$ (and additional thermodynamic quantities) as a function of $\rho$, the temperature $T$, and the electron fraction $Y_{\mathrm{e}}$. Whenever it is necessary in the code to compute the pressure as a function of the specific internal energy $\epsilon$ instead of the temperature $T$, we iterate the corresponding value of $T$ with a Newton-Raphson scheme.

\section{Numerical methods}

Since our new numerical code is based on a previous purely hydrodynamic code (Dimmelmeier et al. 2002a,b) and on its extension to the passive magnetic-field approximation (Cerdá-Durán \& Font 2007; Cerdá-Durán et al. 2007), we describe here in detail only those numerical techniques that represent improvements over their predecessors, and provide only concise information about the numerical schemes already described and tested elsewhere.

The code solves the coupled time evolution of the equations governing the dynamics of the spacetime, the fluid, and the magnetic field in general relativity. The equations are implemented in the code using spherical polar coordinates $\{t, r, \theta, \varphi\}$. We assume axisymmetry and equatorial plane symmetry.

\subsection{Metric solver}

The CFC metric equations, Eqs. (5-6), are five nonlinear elliptic coupled Poisson-like equations, which can be written in compact form as $\hat{\Delta} \boldsymbol{u}(\boldsymbol{x})=\boldsymbol{f}(\boldsymbol{x} ; \boldsymbol{u}(\boldsymbol{x}))$, where $\boldsymbol{u}=u^{k}=\left(\phi, \alpha \phi, \beta^{j}\right)$, and $\boldsymbol{f}=$ $f^{k}$ is the source vector. These five scalar equations are coupled via the source vector, which depends on the components of $\boldsymbol{u}$. We use a fix-point iteration scheme in combination with a linear Poisson solver to solve these equations (for further details see Cerdá-Durán et al. (2005) and Dimmelmeier et al. (2002a)).

Since the CFC equations are written in terms of the energymomentum tensor, the contribution of the energy-momentum of the magnetic field is automatically incorporated in the computations. The main difference with respect to the non-magnetized case arises from the fact that the magnetic-field contribution does not necessarily have compact support. However, the magnetic field far from the fluid should decay at least as a dipole $\left(\sim 1 / r^{3}\right)$, i.e. its contribution can be computed correctly by integrating the $\mathrm{CFC}$ equations in a sufficiently large volume. We checked that in all cases considered here the contribution of the outer magnetic field to the energy-momentum tensor is too small to affect the CFC metric. Hence, we consider the contribution of the magnetic field only up to a radius $\sim 20 \%$ larger than the radial extent of the fluid. The contribution of the inner magnetic field is, however, in some case sufficiently large to modify the metric (e.g. for the magnetized neutron-star equilibria), and therefore cannot be neglected in the CFC equations.

\subsection{Riemann solver}

For the evolution of the matter fields we utilize a HRSC scheme to integrate the subset of equations in the system of Eq. (13) that corresponds to the hydrodynamic variables $\left(D, S_{i}, \tau\right)$. HRSC schemes ensure the numerical conservation of physically conserved quantities and a correct treatment of discontinuities such as shocks (see e.g. Font 2003, for a review and references therein). We implemented various cell-reconstruction procedures that are accurate to either second-order or third-order in space, namely minmod, MC, and PHM (see Toro 1999, for definitions). The time update of the state vector $\boldsymbol{U}$ relies on the method of lines in combination with a second-order accurate Runge-Kutta scheme. The numerical fluxes at cell interfaces are obtained using either the HLL single-state solver of Harten et al. (1983) or the symmetric scheme of Kurganov \& Tadmor (2000) (KT hereafter). Both solvers yield results with an accuracy comparable to Riemann solvers exploiting the full characteristic information, as demonstrated for hydrodynamic special relativistic (Lucas-Serrano et al. 2004) and general relativistic flows in dynamical spacetimes (Shibata \& Font 2005). Tests of both solvers in GRMHD were reported by Antón et al. (2006).

\subsection{Constrained transport scheme}

The evolution of the magnetic field needs to be performed differently from the rest of the conservation equations because the physical meaning of the corresponding conservation equation is different. Although the induction equation can be written in a flux-conservative form, a supplementary condition for the magnetic field (the divergence constraint, or the conservation of the magnetic flux) has to be fulfilled during the whole evolution. Among the numerical schemes that satisfy this condition (see Tóth 2000, for a review), the constrained transport (CT) scheme (Evans \& Hawley 1988) was proven to be adequate for performing accurate simulations of magnetized flows. Our particular implementation of the CT scheme is adapted to the spherical polar coordinates used in the code, and uses cell interface-centered poloidal and (because of the assumption of axisymmetry) cellcentered toroidal magnetic-field components (see Sect.3.2.1 in 
Cerdá-Durán \& Font 2007, for details). The induction equation is discretized in the same way as for the fluid equations.

CT schemes preserve the magnetic flux during the evolution of a magnetized flow, but do not impose the divergence constraint on the initial magnetic field. Hence, one also has to provide initial data that fulfill this constraint in order for the CT method to work properly. This can be ensured by computing the staggered magnetic field from the vector potential (see Eqs. (28) and (29) in Cerdá-Durán \& Font 2007, for details).

Finally, one has to consider the computation of cell-centered values of the (poloidal) magnetic field, which are required in the source terms and for the reconstruction of the magnetic field tangential to the cell interfaces, that enter the evaluation of the numerical flux. Here, we depart from the scheme described by Antón et al. (2006), who computed cell-centered magnetic field components assuming that the corresponding magnetic flux at the cell center is given by the average of the magnetic flux at the cell interfaces. Using this prescription, the cell-centered magnetic pressure differs from that at the interface, even in the case of a homogeneous magnetic field. Instead we use

$B_{i j}^{* r}=-\cos \theta_{i j} \frac{\cos \theta_{j+\frac{1}{2}}-\cos \theta_{j-\frac{1}{2}}}{\sin ^{2} \theta_{j+\frac{1}{2}}-\sin ^{2} \theta_{j-\frac{1}{2}}}\left(B_{i+\frac{1}{2} j}^{* r}+B_{i-\frac{1}{2} j}^{* r}\right)$

$B_{i j}^{* \theta}=\frac{\sin \theta_{i j}}{2}\left(\frac{B_{i j+\frac{1}{2}}^{* \theta}}{\sin \theta_{j+\frac{1}{2}}}+\frac{B_{i j-\frac{1}{2}}^{* \theta}}{\sin \theta_{j-\frac{1}{2}}}\right)$

for the cell-centered magnetic-field components. This prescription guarantees that for a homogeneous field parallel to the rotation axis, the magnetic pressure is equal at the cell center and the cell interface. It also increases the stability of the code for highly magnetized flows, especially near MHD equilibria, and is critical for the success of some of the tests presented here.

\subsection{Recovery of primitive variables}

In relativistic hydrodynamics, in contrast to the Newtonian case, there exists no explicit expression for the primitive variables $\left(\rho, v^{i}, \epsilon\right)$ in terms of the conserved ones $\left(D, S_{i}, \tau\right)$. Hence, a recovery procedure is required whereby the primitive variables are obtained from the conserved ones by inverting the nonlinear system given by Eqs. (10-12) with an efficient numerical algorithm. In most of the recovery algorithms (Noble et al. 2006), one first introduces some scalar quantities, and then solves the resulting simplified system of equations before recovering the primitives.

Following Antón et al. (2006), our recovery procedure is based on the two scalar quantities (note that the first of these is the conserved energy)

$$
\begin{aligned}
\tau= & \rho h W^{2}-P+b^{2}\left(W^{2}-1 / 2\right)-\alpha^{2}\left(b^{0}\right)^{2}-D, \\
S^{2} \equiv & \gamma^{i j} S_{i} S_{j}=\left(\rho h+b^{2}\right)^{2} W^{4} v^{2} \\
& +\alpha^{2}\left(b^{0}\right)^{2}\left[-2 \rho h W^{2}+b^{2}\left(1-2 W^{2}\right)+\alpha^{2}\left(b^{0}\right)^{2}\right] .
\end{aligned}
$$

If one defines $z \equiv \rho h W^{2}$ and makes use of the expression $\boldsymbol{B} \cdot \boldsymbol{S}=\rho h W \alpha b^{0}$ to eliminate $b^{0}$ from these equations, the resulting expressions

$$
\begin{array}{r}
{\left[\left(z+B^{2}\right)^{2}-S^{2}-\frac{2 z+B^{2}}{z^{2}}(\boldsymbol{B} \cdot \boldsymbol{S})^{2}\right] W^{2}-\left(z+B^{2}\right)^{2}=0,} \\
{\left[\tau+D-z-B^{2}+\frac{(\boldsymbol{B} \cdot \boldsymbol{S})^{2}}{2 z^{2}}+P\right] W^{2}+\frac{B^{2}}{2}=0,}
\end{array}
$$

depend only on conserved quantities, on the metric, and on the set of unknowns $\{P, z, W\}$, respectively. The system formed by Eqs. $(21,22)$ and the EOS can then be solved to obtain $\{P, z, W\}$. From these three quantities, the primitive variables can be easily computed as

$$
\begin{aligned}
& \rho=\frac{D}{W}, \\
& v^{i}=\frac{\gamma^{i j} S_{j}+(\boldsymbol{B} \cdot \boldsymbol{S}) B^{i} / z}{z+B^{2}}, \\
& \epsilon=\frac{z-D W-P W^{2}}{D W}
\end{aligned}
$$

The numerical procedure to solve the system of Eqs. $(21,22)$ therefore depends on the EOS (see next two subsections).

\subsubsection{Barotropic fluid}

In a barotropic fluid, the pressure depends only on the density, i.e. $P(\rho)$. In many astrophysical situations (e.g. cold neutron stars) as well as in many standard tests of numerical codes, the fluid is assumed to be barotropic. The most commonly used barotropic EOS is the polytropic EOS, $P=K \rho^{\Gamma}$, where $K$ is the polytropic constant and $\Gamma$ is the adiabatic index.

For a barotropic EOS, the enthalpy is a function of the density only, i.e. $h(\rho)$, and thus $z=D h(\rho) W$. Using this fact and Eq. (23), it is possible to eliminate the unknowns $P$ and $z$ from Eqs. $(21,22)$. The Lorentz factor $W$ then remains to be computed numerically by solving one of these equations.

Following Antón (2007), we solve Eq. (21) for $W$ by means of the bisection method, and then recover $P$ and $z$ using the EOS. This method is extremely robust and always leads to a solution for $W$, provided that it lies between the initial lower and upper guess value.

\subsubsection{Baroclinic fluid}

This is the most common form of the EOS in hydrodynamic simulations because it takes into account temperature effects. We implemented several baroclinic EOS in our numerical code, namely the ideal gas EOS, $P=\rho \epsilon(\Gamma-1)$, the analytic hybrid EOS (Janka et al. 1993), and a tabulated microphysical EOS.

Irrespective of the baroclinic EOS used, it can always be expressed in the form $P\left(\rho, \epsilon, Y_{i}\right)$. Since the composition $Y_{i}$ (the index $i$ runs over all relevant species) is known directly from the hydrodynamics, the dependence of the EOS on the composition does not affect the recovery procedure. The following discussion therefore can be restricted to an EOS of the form $P(\rho, \epsilon)$.

For a baroclinic fluid, the system formed by Eqs. (21-22) and the EOS expressed as

$P-P(\rho, \epsilon)=0$

must be solved numerically. In general, it is not possible to use the EOS to analytically remove the dependence of Eqs. $(21,22)$ on $P$, since $\epsilon$ depends on the pressure itself due to Eq. (25). However, for some analytic EOS, e.g. an ideal gas, Eqs. (23) and (25) can be used to express the pressure as a function of $z$ and $W$ only. This allows one to eliminate $P$ from Eqs. $(21,22)$, reducing the system to be solved to two equations with the unknowns $z$ and $W$. Since the numerical method should not rely on any assumption about the EOS, we do not exploit this simplification. Instead we solve the system of Eqs. $(21,22,26)$ by means of a Newton-Raphson scheme, which converges rapidly 
provided the initial guess is sufficiently good (see below). We consider the Newton-Raphson iteration to be converged when the relative error of the variables is less than a certain tolerance (typically $10^{-12}$ ).

Microphysical tabulated EOS. Some of the equations of state available from nuclear physics that are used in astrophysics are not provided in terms of the specific internal energy. In general, the EOS depends on the composition of the fluid (usually the electron fraction $Y_{\mathrm{e}}$ ) and on the temperature $T$ instead of $\epsilon$. Hence, one has to deal with tabulated EOS of the form

$P=P\left(\rho, T, Y_{\mathrm{e}}\right)$,

$\epsilon=\epsilon\left(\rho, T, Y_{\mathrm{e}}\right)$.

A first approach to handle such an EOS is to obtain effectively $P=P\left(\rho, \epsilon, Y_{\mathrm{e}}\right)$ by computing the value of $T$ that satisfies Eq. (28) for a given value of $\epsilon$. The procedure described above for an EOS of the form $P(\rho, \epsilon)$ can then be applied, since $Y_{\mathrm{e}}$ is known directly from the evolution. This approach was successfully used in the hydrodynamic simulations presented by Ott et al. (2007b). In the magnetized case, however, we find this approach to be problematic for strong magnetic fields $\left(P_{\text {mag }} / P>1\right)$. The solver is able to recover the exact value in that regime only if the initial guess is very close to the solution, which renders the code unstable. To avoid this problem we add the equation

$\epsilon-\epsilon\left(\rho, T, Y_{\mathrm{e}}\right)=0$,

to the Newton-Raphson system, and solve the extended system of Eqs. $(21,22,29)$ for the unknowns $z, W$, and $T$. This allows one to use directly the EOS as a function of $T$ instead of $\epsilon$. We find that this method is very stable and has a much larger radius of convergence than the first approach (see Sect. 4.1).

"Safe" guess values. When we use the values of the previous time step as an initial guess for the Newton-Raphson iteration at a given time step, the solver usually converges within a few iterations. However, sometimes the guess values are too far away from the solution, and the Newton-Raphson iteration fails. In such a case, we restart the iteration process using a "safe" set of guess values, which we choose to be upper limits to the unknowns $\{P, z, W\}$ (or $\{T, z, W\}$ ). This choice leads to a rather robust recovery scheme as demonstrated by our test calculations (see Sect. 4).

To derive an upper limit for $z$, we define $\delta$ as the angle between $\boldsymbol{v}$ and $\boldsymbol{B}$, i.e. $\boldsymbol{v} \cdot \boldsymbol{B}=\sqrt{\boldsymbol{v}^{2} \boldsymbol{B}^{2}} \cos \delta$. Using this angle, we have

$$
\begin{aligned}
\alpha^{2}\left(b^{0}\right)^{2} & =W^{2} \boldsymbol{B}^{2} \boldsymbol{v}^{2} \cos ^{2} \delta, \\
b^{2} & =\frac{\boldsymbol{B}^{2}}{W^{2}}\left(1+\left(W^{2}-1\right) \cos ^{2} \delta\right) .
\end{aligned}
$$

From the definition of $\tau$ given in Eq. (12), one obtains $z$ as

$z=\tau+P+D-\frac{B^{2}}{2}-\frac{B^{2}}{2} \frac{W^{2}-1}{W^{2}}\left(2-\cos ^{2} \delta\right)$.

Since the last term in this equation is always negative or zero, an upper limit for $z$ is given by

$z \leq \tau+P+D-\frac{B^{2}}{2}$
However, this upper limit cannot be computed directly from the conserved quantities, since the pressure is unknown. Hence, we first need to determine an upper limit for the pressure. If we assume that the pressure grows monotonically with $\rho$ and $\epsilon$, which is a reasonable assumption for the types of EOS that we use in the code, then we only need to derive upper limits for $\rho$ and $\epsilon$, and hence

$P \leq P_{\max } \equiv P\left(\rho_{\max }, \epsilon_{\max }\right)$.

It is easy to find an upper limit for $\rho$, since $W \geq 1$,

$\rho \leq \rho_{\max } \equiv D$.

In the case of the specific internal energy, we substitute Eq. (32) into Eq. (25) and obtain

$$
\begin{gathered}
\epsilon=\frac{1}{D W}\left[\tau-\frac{B^{2}}{2}+P\left(1-W^{2}\right)+D(1-W)\right. \\
\left.-\frac{B^{2}}{2} \frac{W^{2}-1}{W^{2}}\left(2-\cos ^{2} \delta\right)\right] .
\end{gathered}
$$

Using again the fact that $W \geq 1$ we derive the upper limit

$\epsilon \leq \epsilon_{\max } \equiv \frac{1}{D}\left[\tau-\frac{B^{2}}{2}\right]$.

An upper limit for $z$ is given by

$z \leq z_{\max } \equiv \tau+P_{\max }+D-\frac{B^{2}}{2}$

which coincides with $z$ in the limit of small velocities, $W \rightarrow 1$.

We were unable to compute an analytic upper limit for $W$; however it is easy to set an upper limit from physical considerations. In the core-collapse simulations in which we are interested, the Lorentz factor is not expected to exceed a value of 10 . Nevertheless, we chose a much larger guess value for $W$, since the number of iterations until convergence is very insensitive to the precise value of the upper limit. Accordingly, the "safe" guess values that we use in the Newton-Raphson solver are

$P_{\text {guess }}=P_{\max }$,

$z_{\text {guess }}=z_{\max }$,

$W_{\text {guess }}=10000$,

$T_{\text {guess }}=T\left(\rho_{\max }, \epsilon_{\max }\right)$.

We note that $T_{\text {guess }}$ is not an upper limit for the temperature in general, but the pressure value computed with $T_{\text {guess }}$ and $\rho_{\max }$ provides an upper limit for the pressure, i.e. $P \leq P\left(\rho_{\text {max }}, T_{\text {guess }}\right)$.

\subsection{Vacuum treatment}

The presence of vacuum regions is common in numerical simulations dealing with astrophysical scenarios. These regions are usually avoided by imposing a numerical atmosphere surrounding the object under study, i.e. a small floor value for the rest mass density, which allows one to use the same recovery procedure in regions filled with the numerical atmosphere and the fluid. A vacuum region would cause the recovery procedure to fail both in the hydrodynamic and in the magneto-hydrodynamic case, as can be inferred from Eqs. $(24,25)$. The numerical atmosphere approach is commonly used in hydrodynamic simulations (see e.g. Font et al. 2002; Dimmelmeier et al. 2002a) as well as in GRMHD simulations (Duez et al. 2005; Shibata \& Sekiguchi 2005; Giacomazzo \& Rezzolla 2007). 
In the unmagnetized case, the floor value of the numerical atmosphere is chosen such that it does not affect significantly the dynamics of the system. This can be achieved by choosing the threshold value for the rest mass density to be a small fraction of the maximum density in the initial model, typically $\rho_{\text {thr }} \approx$ $10^{-6} \rho_{\text {max }}$. Every grid point with $\rho<\rho_{\text {thr }}$ is reset to the numerical atmosphere value, i.e. $\rho=\rho_{\text {atm }}$ and $v^{i}=0$, where the floor value for the rest mass density is $\rho_{\mathrm{atm}} \approx 10^{-3} \rho_{\mathrm{thr}}$.

In the magnetized case additional problems arise. Since the transition to the numerical atmosphere usually results in a steep profile in $\rho$ (which drops to the floor value) but not necessary in $B$ (magnetic field lines can extend into the vacuum), atmosphere regions can easily have large ratios of $P_{\mathrm{mag}} / P$, even if the fluid is weakly magnetized. This problem increases as the floor value $\rho_{\text {atm }}$ is reduced, and can lead to problems with the recovery of the primitive variables in the atmosphere. To avoid this problem, some authors (Duez et al. 2005; Shibata \& Sekiguchi 2005) do not allow magnetic fields in the numerical atmosphere by choosing magnetic fields confined to the fluid regions. Other authors (Giacomazzo \& Rezzolla 2007; Shibata et al. 2007) apply a floor to the hydrodynamic variables and allow the magnetic field to evolve freely. This approach works fine, if the ratio of $P_{\mathrm{mag}} / P$ does not exceed the critical value above which the recovery procedure fails. We estimate this critical value for our code in Sect. 4.1. Consequently, a sufficiently low density atmosphere will show the correct dynamic behavior when the magnetic field strength in the atmosphere is limited. On the other hand, if one wishes to simulate stronger magnetic fields, one must use a denser atmosphere that can even affect the dynamics of the system (Shibata et al. 2007).

We also allow for a freely evolving magnetic field in the atmosphere, since we are then not restricted to any particular magnetic field structure. To overcome the problem of the high magnetization $P_{\mathrm{mag}} / P$ in the atmosphere, we choose compromise values for $\rho_{\text {atm }}$ depending on the problem to be solved. Since there are cases (e.g.for the evolution of neutron stars in Sect. $4.3, P_{\mathrm{mag}} / P$ becomes as large as $10^{13}$ ) where the ratio $P_{\mathrm{mag}} / P$ exceeds the critical value for the recovery procedure $\left(10^{6}-10^{8}\right.$; see Sect. 4.1) even for reasonable values of $\rho_{\text {atm }}$, we use a fast atmosphere checking routine which avoids the recovery of the primitives in the respective zones.

If we are able to mark a zone as being part of the atmosphere before the recovery of the primitives is performed, we can avoid the recovery because the values for the primitives in these zones are set to the floor value. Since $D \geq \rho$, if $D<\rho_{\text {thr }}$ holds, then $\rho<\rho_{\text {thr }}$, and the zone is part of the atmosphere. Hence, we can use this condition to check whether a zone belongs to the atmosphere before performing the recovery. We note that for atmosphere zones, whose velocities are set to zero at every time step, it is very unlikely that the value of the (unknown) Lorentz factor $W$ at the next time step differs significantly from 1 , i.e. $\rho \approx D$ in these zones. Using this procedure, we can handle arbitrarily large magnetic fields in the atmosphere without imposing any limitation on the value of $\rho_{\mathrm{atm}}$.

In the equilibrium models of magnetized neutron stars (Sect. 4.3), we keep the magnetic field fixed in the atmosphere to its initial value. This is a reasonable choice since for an equilibrium configuration the outside magnetic field should not change during the evolution. The advantage of this approach is that the time step is not dominated by the atmosphere where the eigenvalues are close to the speed of light, but by the neutron star interior with eigenvalues of the order of $\approx 0.1$. This results in a speed-up of about a factor of 10 in these computations. We note that this speed-up is only possible because in the CFC
Table 1. Test of the recovery of the primitive variables.

\begin{tabular}{llccc}
\hline \hline Test & EOS & $\rho\left[\mathrm{g} \mathrm{cm}^{-3}\right]$ & $\epsilon$ & $Y_{\mathrm{e}}$ \\
\hline PN & Polytropic & $3 \times 10^{14}$ & - & - \\
PE & Polytropic & $10^{12}$ & - & - \\
HN1 & Hybrid & $3 \times 10^{14}$ & 0.001 & - \\
HN2 & Hybrid & $3 \times 10^{14}$ & 0.01 & - \\
HN3 & Hybrid & $3 \times 10^{14}$ & 0.1 & - \\
HN4 & Hybrid & $3 \times 10^{14}$ & 1.0 & - \\
HE1 & Hybrid & $10^{12}$ & 0.001 & - \\
HE2 & Hybrid & $10^{12}$ & 0.01 & - \\
HE3 & Hybrid & $10^{12}$ & 0.1 & - \\
HE4 & Hybrid & $10^{12}$ & 1.0 & - \\
I1 & Ideal gas & $10^{14}$ & 0.01 & - \\
I2 & Ideal gas & $10^{14}$ & 0.1 & - \\
I3 & Ideal gas & $10^{14}$ & 1.0 & - \\
I4 & Ideal gas & $10^{14}$ & 10.0 & - \\
I5 & Ideal gas & $10^{14}$ & 100.0 & - \\
I6 & Ideal gas & $10^{14}$ & 1000.0 & - \\
I7 & Ideal gas & $10^{14}$ & 10000.0 & - \\
S1 & SHEN & $2.4 \times 10^{14}$ & 0.055 & 0.25 \\
S2 & SHEN & $4.2 \times 10^{9}$ & 0.009 & 0.427 \\
S3 & SHEN & $4.2 \times 10^{8}$ & 0.008 & 0.457 \\
S4 & SHEN & $2.6 \times 10^{6}$ & 0.009 & 0.5 \\
\hline
\end{tabular}

Varying the flow velocity $\boldsymbol{v}$, the magnetic field $\boldsymbol{B}$, and the angle between $\boldsymbol{B}$ and $\boldsymbol{v}$ in a wide range, the recovery procedure is tested for the equations of state, the densities $\rho$, the specific internal energies $\epsilon$, and the electron fractions $Y_{\mathrm{e}}$ given in Cols. 2 to 5, respectively.

approximation the metric evolution does not constrain the size of the time step. In codes based on hyperbolic formulations of the Einstein equations, the time step is always limited by the lightcrossing time of the zones, i.e. this speed-up is impossible. All other existing GRMHD codes with dynamic spacetimes (Duez et al. 2005; Shibata \& Sekiguchi 2005; Giacomazzo \& Rezzolla 2007) suffer from this limitation.

\section{Code tests}

\subsection{Recovery of the primitive variables}

The numerical method used for the recovery of the primitive variables $\left\{\rho, v^{i}, \epsilon, B^{j}\right\}$ from the conserved ones $\left\{D, S_{i}, \tau, B^{j}\right\}$, is tested by varying the flow velocity $\boldsymbol{v}$, the magnetic field $\boldsymbol{B}$, and the angle $\delta$ between $\boldsymbol{B}$ and $\boldsymbol{v}$ over a wide range. Instead of varying $\boldsymbol{v}$ and $\boldsymbol{B}$ we vary the Lorentz factor $W-1$ in the interval $\left[10^{-4}, 10^{4}\right]$, and the magnetization $P_{\mathrm{mag}} / P$ in the interval $\left[10^{-8}, 10^{10}\right]$, respectively. We choose values of $\rho, \epsilon$, and (for the tabulated EOS only) $Y_{\mathrm{e}}$ that are typical for core collapse (Table 1). Test cases PN and PE correspond to a polytropic EOS with $\Gamma=2, K=1.455 \times 10^{5}$ (cgs units), and $\Gamma=4 / 3$, $K=4.897 \times 10^{14}$, respectively. For the hybrid EOS, the test cases HN and HE probe densities above and below nuclear matter density, while the SHEN EOS cases test the typical conditions inside a proto-neutron star (S1) and the progenitor core (S2 to $\mathrm{S} 4$ ).

For a baroclinic EOS, we use the "safe" values given in Sect. 3.4.2 as guess values for the Newton-Raphson iteration, and choose a tolerance of $10^{-12}$. Figure 1 shows the region in which the recovery scheme converges well, i.e. where the relative difference between the values of the recovered primitive variables and their exact values is less than $10^{-10}$. For all considered EOS, the parameter space of astrophysical interest is well 

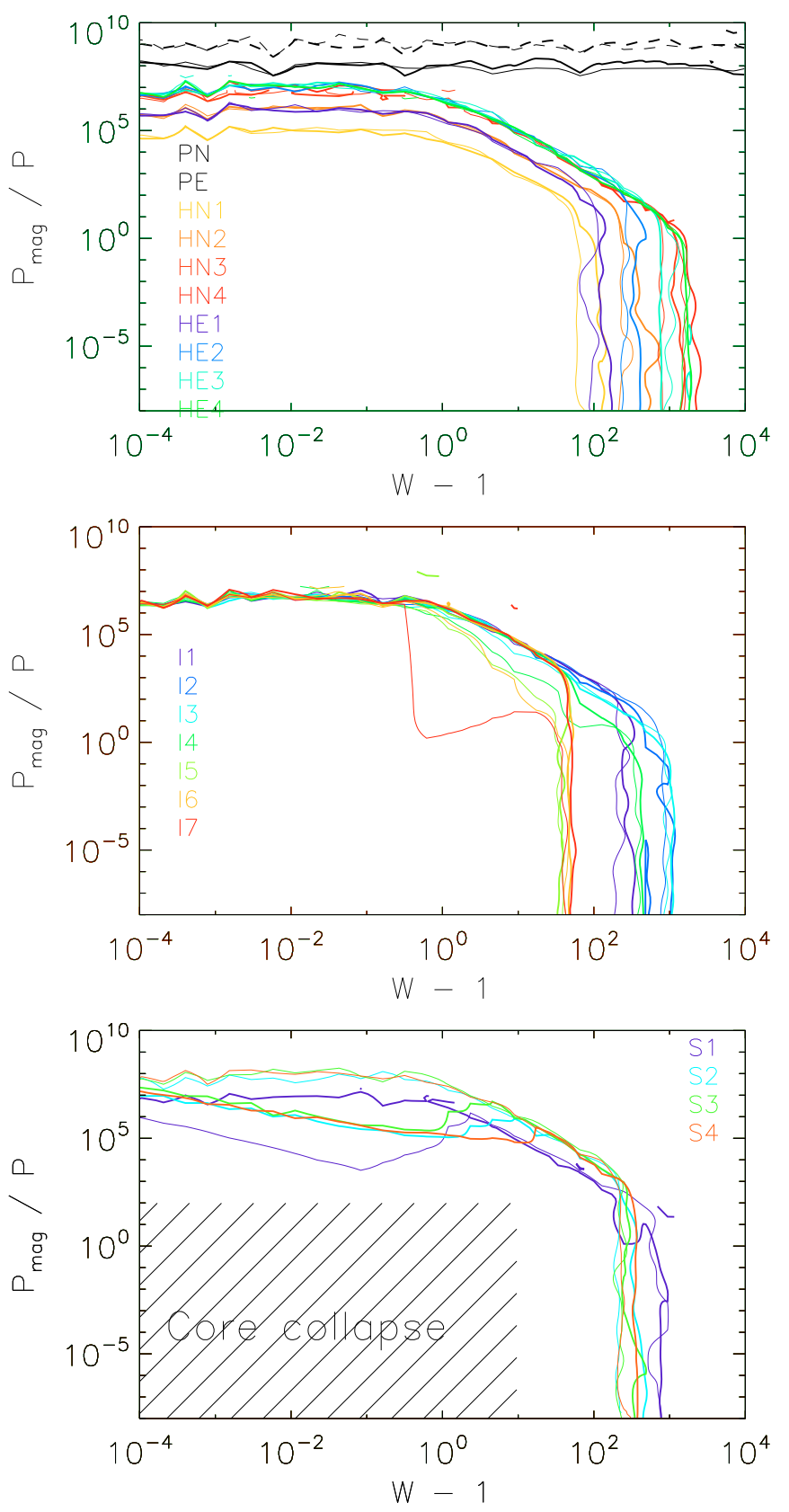

Fig. 1. Upper limits for the Lorentz factor $(W-1)$ and the magnetization $P_{\text {mag }} / P$ for which the relative difference between the values of the recovered primitive variables and their exact values is less than $10^{-10}$. The upper panel shows these limits for the polytropic and the hybrid EOS, the middle panel for the ideal gas EOS, and the lower panel for the tabulated SHEN EOS, respectively. Thin lines correspond to the case $\cos ^{2} \delta=0$, and thick lines to $\cos ^{2} \delta=1.0$. The shaded region in the bottom panel shows the typical parameter space encountered in corecollapse simulations.

covered. In test case I7, the region of convergence is reduced substantially when $\cos \delta=0$. This test, that corresponds to an extreme ideal gas with $\epsilon=10^{4}$, was chosen to determine the maximum value of $\epsilon$ which can be recovered and lies outside the parameter range of interest in core collapse. We also determine upper limits to the magnetization $P_{\mathrm{mag}} / P$ in the low velocity limit ranging from values of $10^{6}$ to $10^{8}$. In the low magnetic field limit the maximum Lorentz factor that the recovery procedure can handle is $10^{2}$ to $10^{3}$. If any of these limits is exceeded, the numerical scheme is unable to recover the primitive quantities within the required accuracy. The reason for the recovery failure in the three limiting cases for $\epsilon, W$, and $P_{\mathrm{mag}} / P$ is that the contribution of internal energy, kinetic energy or magnetic energy, respectively, is dominant in the system and any other kind of energy has a very small contribution. In these cases large changes in the subdominant terms will produce small changes in the recovery equations and the system may therefore converge to a wrong solution within a given accuracy. If the tolerance value is reduced, these limits can be extended. We note that in astrophysical situations involving baryonic matter, it is unlikely to encounter values of $\epsilon>1, W>10$, or $P_{\text {mag }} / P>100$. Therefore, we consider that our recovery procedure is sufficiently robust for simulations of core collapse and involving compact objects.

Using the "safe" guess values, the number of iterations needed for the Newton-Raphson solver to converge is relatively large: 50-70 for both the hybrid and the ideal gas EOS, and 50-200 for the tabulated EOS. However, during a numerical simulation, the "safe" guess is only used if the regular guess (the value from the previous time step) fails. If we use guess values that differ by only $10 \%$ from the exact ones, the NewtonRaphson converges more rapidly, within 10-20 iterations for the hybrid and ideal gas EOS, and 20-30 for the tabulated EOS. For the polytropic EOS it takes about $40-60$ bisection steps to achieve the required tolerance.

\subsection{Spherical explosion}

Since the majority of existing (2D) MHD codes are written in cylindrical coordinates, a commonly performed test is the simulation of a cylindrical explosion. For relativistic MHD codes, such a setup was proposed by Komissarov (1999), which was also used by other authors (Del Zanna et al. 2003; Leismann et al. 2005). However, when using a code based on spherical coordinates the most natural choice is a spherical explosion. Kössl et al. (1990) performed this test with a Newtonian MHD code, but to the best of our knowledge no spherical explosions test has been performed in relativistic MHD. Therefore, we consider here a spherical explosion test for which the initial jump conditions are identical to those of the cylindrical test of Komissarov (1999).

Our test setup consists of an initial radially symmetric explosion zone $(r<1)$ surrounded by a highly magnetized ambient gas for $r>1$. In the outer part of the explosion region $(0.8<r<1.0)$, we set the state variables decline exponentially to the values of the ambient medium (Table 2). The velocity is initially zero everywhere, and the magnetic field is homogeneous and parallel to the symmetry axis. The background spacetime is assumed to be flat. The initial data are evolved using an ideal gas EOS with an adiabatic index $\Gamma=4 / 3$. The computational grid is evenly spaced in radius and angle, and extends in the radial direction up to a maximum radius of $r=6.0$. We perform the test with two $(r, \theta)$ resolutions $(80 \times 20$, and $160 \times 40)$ for all reconstruction schemes and flux formulae.

Table 2 shows the eigenvalue structure of the initial setup. Since the explosion region is weakly magnetized $\left(P_{\mathrm{mag}} / P=\right.$ $\left.5 \times 10^{-3}\right)$, the dominant wave in this region is the fast magnetosonic wave, which propagates at a speed $\left|\lambda_{f}\right|$ close to that of the corresponding hydrodynamic wave. All other wave speeds are close to zero, i.e. the inner region will expand with the velocity $\left|\lambda_{f}\right|$. The ambient gas is highly magnetized, and the full wave structure is significant there. The only waves fast enough to travel ahead of the explosion shock are the fast magnetosonic 
Table 2. Spherical explosion test.

\begin{tabular}{llllllllll}
\hline \hline & $P$ & $\rho$ & $|\boldsymbol{B}|$ & $P_{\mathrm{mag}} / P$ & $\left|\lambda_{f \pm}\right|$ & $\left|\lambda_{\mathrm{A} \pm}\right|$ & $\left|\lambda_{s \pm}\right|$ & $\left|\lambda_{\mathrm{e}}\right|$ & $\left|\lambda_{ \pm}\right|$ \\
\hline$r<1$ & 1 & $10^{-2}$ & 0.1 & $5 \times 10^{-3}$ & $0.578,0.576$ & $0,0.05$ & $0,0.05$ & 0 & 0.576 \\
$r>1$ & $3 \times 10^{-5}$ & $10^{-4}$ & 0.1 & 166.6 & $0.991,0.989$ & $0,0.988$ & $0,0.426$ & 0 & 0.426 \\
\hline
\end{tabular}

Initial values for the pressure $P$, the density $\rho$, the magnetic field $|\boldsymbol{B}|$, and the magnetization $P_{\mathrm{mag}} / P$ are given in Cols. 2 to 5 for the explosion region $(r<1)$ and in the ambient region $(r>1)$, respectively. The eigenvalues, namely the speeds of the fast magnetosonic wave $\lambda_{f \pm}$, the Alfvén wave $\lambda_{\mathrm{A} \pm}$, the slow magnetosonic wave $\lambda_{s \pm}$, and of the entropy wave $\lambda_{\mathrm{e}}$ in the radial direction at the initial time are given in Cols. 6 to 9 , both for the equator (left value) and the pole (right value). In addition, we provide in the last column the value of the eigenvalue (sound speed) $\lambda_{ \pm}$of the corresponding non-magnetized case.
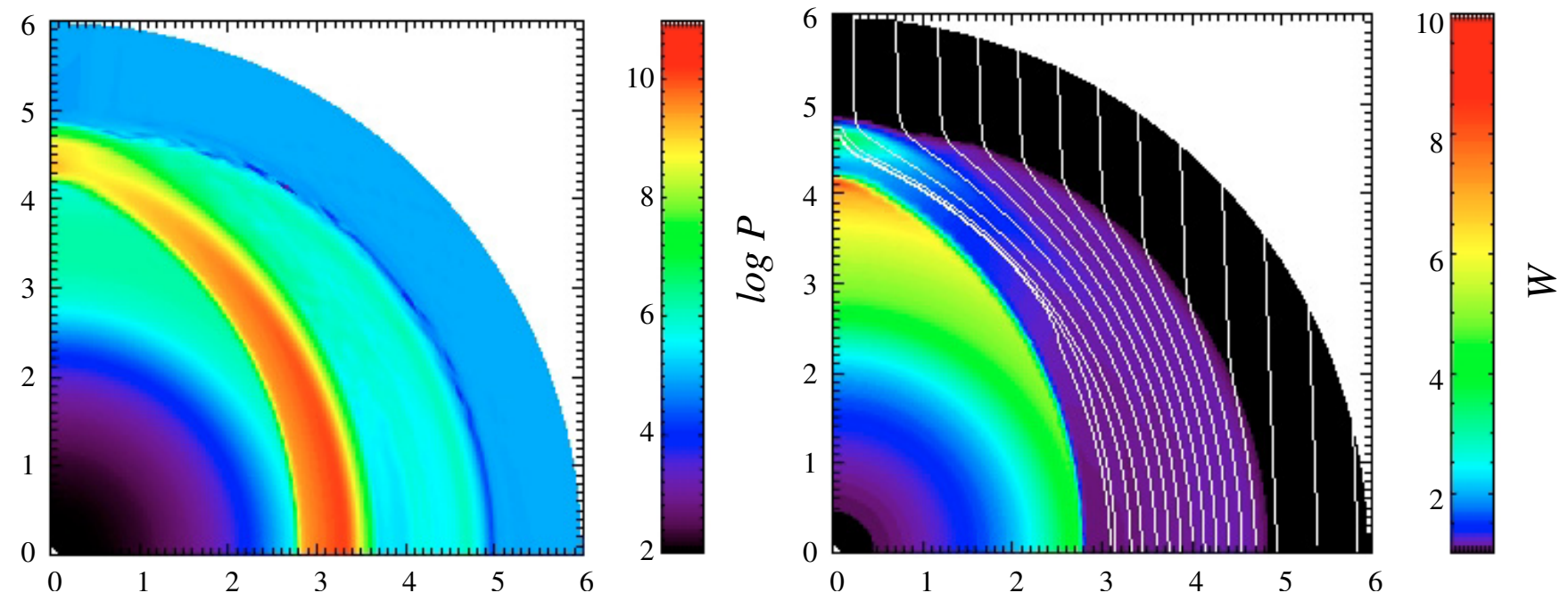

Fig. 2. Snapshot of the spherical explosion test at $t=4$. The panels show the logarithm of the pressure (left) and Lorentz factor, and the magnetic field lines (right). The simulation was performed with $160 \times 40$ zones using PHM cell reconstruction and the KT flux formula.

wave, which propagates with an almost angular-independent radial velocity close to the speed of light, and the Alfvén wave, whose radial velocity is also close to $c$ along the symmetry axis, but varies as $\cos \theta$.

Figure 2 shows a snapshot at the end of the simulation $(t=4)$. Both the Lorentz factor and the pressure distribution clearly show the wave structure mentioned above. The spherical fast magnetosonic wave is located at $r \approx 5$, and the trailing strong shock, which is deformed due to the magnetic field, consists of a mixture of the bulk expansion of the inner region and an Alfvén wave propagating faster along the axis. Even further inwards, a rarefaction wave is visible, which is almost spherically symmetric since the magnetization in this region is rather low.

The corresponding radial profiles of $P$ and $W$ both along the equator (upper panels) and the axis (lower panels) are displayed in Fig. 3 for various numerical methods. These plots are qualitatively similar to those of the cylindrical explosion test (see e.g. Fig. B.4 in Leismann et al. 2005). All numerical schemes exhibit first order convergence as expected for flows with shocks. The MC and PHM schemes yield very similar results, while the minmod scheme gives slightly smaller values. No significant differences are found between the results obtained using the HLLE and KT flux formulae.

\subsection{Magnetized neutron stars}

The previous two tests demonstrate the ability of the code to handle extreme situations such as high magnetization, large Lorentz factors, and strong shocks. In this section we show its correct behavior in curved spacetimes, particularly in dynamic ones.
An astrophysical scenario that can be used for this assessment is the evolution of equilibrium neutron stars, a test which is frequently used for general relativistic hydrodynamics codes (Shibata 1999; Font et al. 2002; Dimmelmeier et al. 2002a; Duez et al. 2003; Cerdá-Durán et al. 2005) as well as for GRMHD codes (Giacomazzo \& Rezzolla 2007). Of all presently existing codes capable of solving the GRMHD equations coupled to a dynamic spacetime (Duez et al. 2005; Shibata \& Sekiguchi 2005; Giacomazzo \& Rezzolla 2007; Anderson et al. 2008), this demanding test, involving all aspects of the code and in particular the correct coupling between metric and MHD equations, has only been performed by the code of Giacomazzo \& Rezzolla (2007).

As initial models for the magnetized neutron star test, we use the relativistic self-consistent equilibrium models of Bocquet et al. (1995), where all effects of the magnetic field (Lorentz force, spacetime curvature generated by the magnetic contribution to the energy-momentum tensor) are taken into account. The equilibrium models are computed using the LORENE library ${ }^{1}$. We construct non-rotating polytropic equilibrium models with $\Gamma=2$ and $K=1.455 \times 10^{5}$ (cgs units). The central enthalpy is chosen to be $\ln h_{\mathrm{c}}=0.228$, and the magnetic field is that of a perfect conductor with the current density of Bocquet et al. (1995) and vacuum outside. By increasing the value of the central current density $j_{0}$ from 0 to $5 \times 10^{14} \mathrm{~A} \mathrm{~m}^{-2}$, we compute a sequence of equilibrium models with a magnetic field ranging from zero to $1.8 \times 10^{16} \sqrt{4 \pi}$ Gauss (Table 3 ). The magnetic field topology is shown in Fig. 4 for a representative model (MNS3). It is purely poloidal with field lines crossing the surface of the neutron star

\footnotetext{
${ }^{1}$ http://www. lorene.obspm.fr/
} 

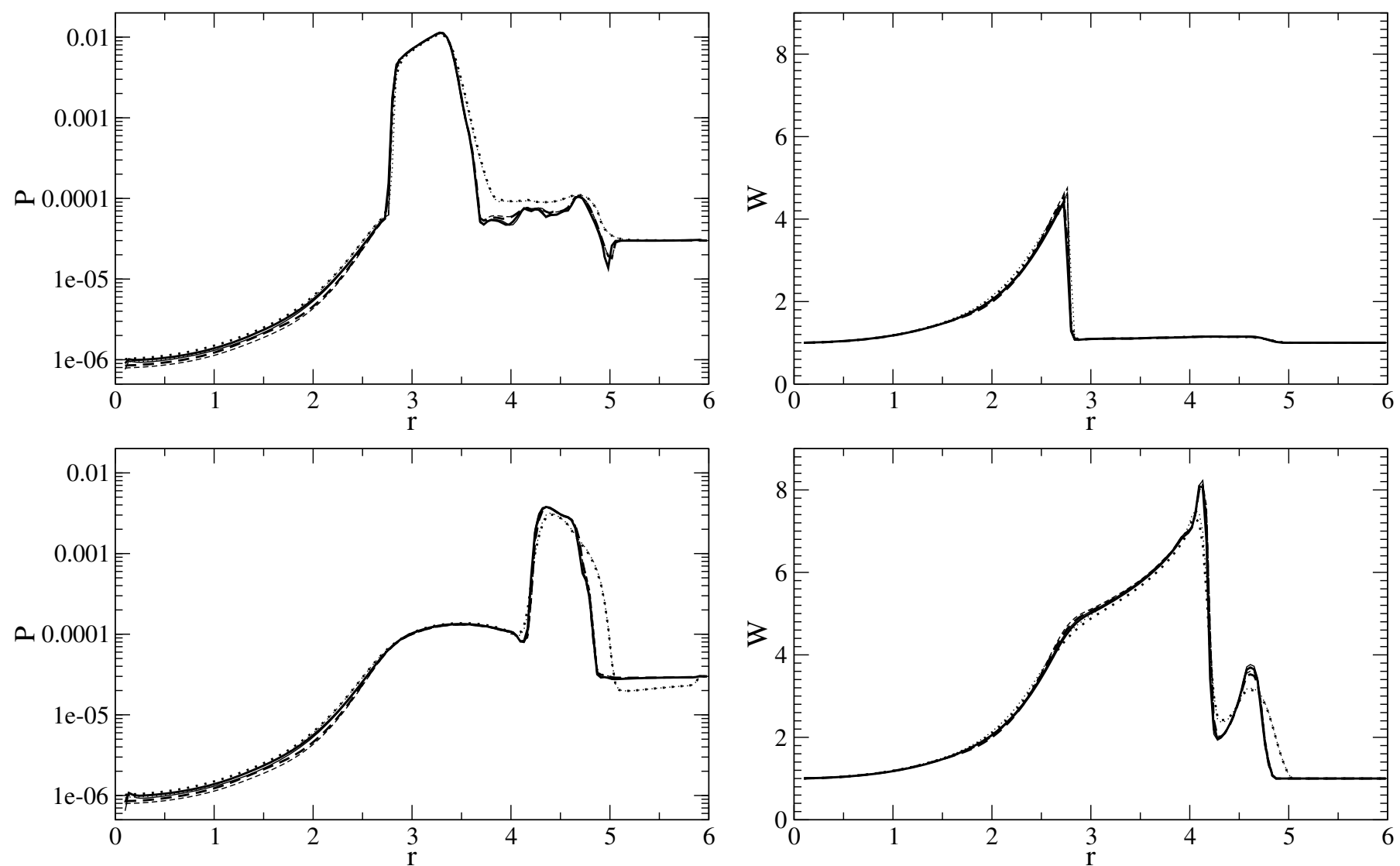

Fig. 3. Results for the spherical test explosion at $t=4$. The panels show radial profiles of the fluid pressure $P$ (left panels) and the Lorentz factor $W$ (right panels) along the equator (upper panels) and the polar axis (lower panels), respectively. The lines styles differentiate between the reconstruction schemes: minmod (dotted), MC (dashed), and PHM (solid). Results for two different flux formulae are shown: HLLE (thin lines) and KT (thick lines). Note that the results obtained with these two flux formulae are often so similar that they cannot be distinguished. The grid resolution used is $160 \times 40$ zones.

Table 3. Initial models of magnetized neutron stars.

\begin{tabular}{ccccccc}
\hline \hline Model & $j_{0}\left[A m^{-2}\right]$ & $r_{\mathrm{e}}[\mathrm{km}]$ & $r_{\mathrm{p}} / r_{\mathrm{e}}$ & $P_{\mathrm{mag}} /\left.P\right|_{\mathrm{c}}$ & $|\boldsymbol{B}|_{\mathrm{c}}[\sqrt{4 \pi} \mathrm{G}]$ & $M_{\mathrm{ADM}}\left[M_{\odot}\right]$ \\
\hline MNS0 & 0 & 11.998 & 1.0 & & 0 & 1.40 \\
MNS1 & $2 \times 10^{13}$ & 11.998 & 0.999992 & $5.75 \times 10^{-6}$ & $7.2 \times 10^{14}$ & 1.40 \\
MNS2 & $2 \times 10^{14}$ & 11.999 & 0.9992 & $5.76 \times 10^{-4}$ & $7.2 \times 10^{15}$ & 1.40 \\
MNS3 & $5 \times 10^{14}$ & 12.006 & 0.995 & $3.63 \times 10^{-3}$ & $1.8 \times 10^{16}$ & 1.40 \\
\hline
\end{tabular}

From left to right, the columns give the central current density $j_{0}$, the equatorial radius $r_{\mathrm{e}}$, the ratio of polar to equatorial radius $r_{\mathrm{p}} / r_{\mathrm{e}}$, the ratio of magnetic to thermal pressure $P_{\mathrm{mag}} / P$ at the center of the star, the central magnetic field $|\boldsymbol{B}|_{\mathrm{c}}$, and the ADM mass $M_{\mathrm{ADM}}$ of each model, respectively.

(thick dash-dotted line). At sufficiently large distances from the star, the magnetic field has a dipole topology.

First, we perform simulations in the Cowling approximation, where the spacetime is kept fixed. We stop the evolution after $5 \mathrm{~ms}$ which corresponds to $52 t_{\mathrm{dyn}}$, where $t_{\mathrm{dyn}}=\sqrt{r_{\mathrm{e}}^{3} / M}$ is the characteristic dynamic time-scale of the system. Using the Cowling approximation, allows us to test the behavior of our MHD scheme without including yet the coupled evolution of the spacetime itself. The spacetime fields are computed using the CFC equations in the first time step, and their values are kept fixed afterwards. To carry out convergence tests, we performed computations with models MNS0 and MNS3 on equidistant grids $\left(n_{r} \times n_{\theta}\right)$ with $80 \times 10,160 \times 20$, and $320 \times 40$ zones, respectively. The other two models, MNS1 and MNS2, were simulated only with $160 \times 20$ zones. We use the PHM reconstruction scheme and the KT flux formula in all computations reported in this section. The neutron star is surrounded by an atmosphere as described in Sect. 3.5 with a threshold value of $\rho_{\text {thr }}=10^{-7} \rho_{\max }$, and a floor value $\rho_{\text {atm }}=10^{-9} \rho_{\max }$. In the highly magnetized models MNS2 and MNS3 the value of $P_{\text {mag }} / P$ is close to the critical value for the recovery procedure in the outermost zone of the neutron star. In these models, we raise the threshold value to $\rho_{\text {thr }}=10^{-6} \rho_{\text {max }}$ keeping the same floor value.

Three of the panels of Fig. 5 show the evolution of the central density with time. Due to numerical truncation errors in the remapping of the equilibrium model from the spectral grid used by LORENE to our finite-difference grid, some small amplitude perturbations are triggered, which excite the normal modes of pulsation of the star. This causes the periodic oscillations of the central density. The neutron star remains in equilibrium throughout its evolution, only a small drift with time is visible in the central density evolution. As we increase the grid 


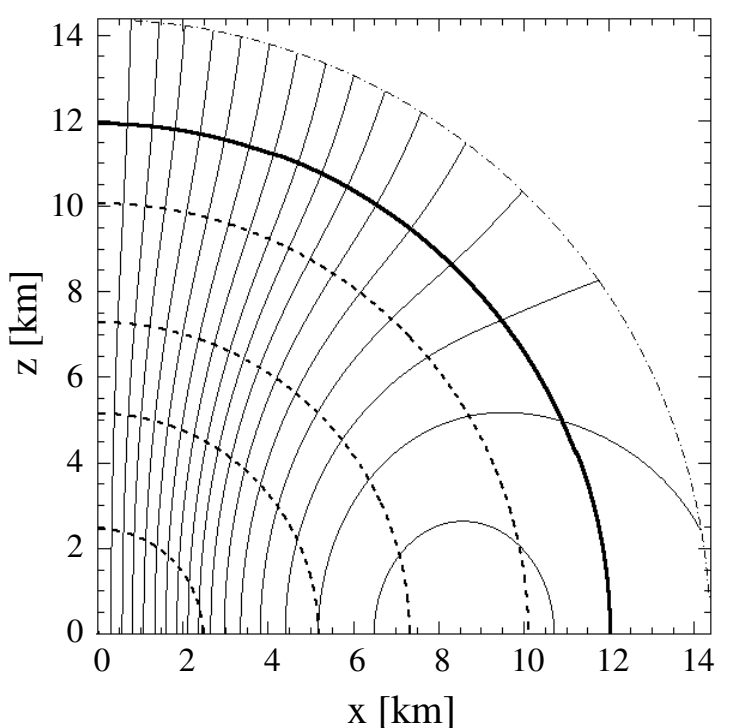

Fig. 4. Magnetic field lines structure of model MNS3. The thin solid lines represent the magnetic field lines while the thick dashed lines are rest mass density isocontours for $1,3,5$ and $7 \times 10^{14} \mathrm{~g} \mathrm{~cm}^{-3}$. Moreover, the thick solid line represents the surface of the star, and the thin dashdotted line marks the boundary of the numerical grid.

resolution, this drift tends to zero. Comparing the nonmagnetized model (MNS0) with the magnetized models (MNS1, MNS2, and MNS3), the drift, although small $(<0.2 \%$ in the $160 \times 20$ models), is larger in the magnetized models (lower left panel of Fig. 5). We find that the drift is very sensitive to the value of $\rho_{\mathrm{thr}}$ in model MNS0, if $\rho_{\mathrm{thr}}>10^{-5} \rho_{\max }$ (for smaller values, there is no influence). We suppose that, for denser atmospheres there, is a coupling between the star and the atmosphere that allows a transfer of mass and momentum from the interior to the atmosphere (see the related discussion in Stergioulas et al. 2004, and Dimmelmeier et al. 2006). This has two consequences for the evolution: first, the oscillations are damped more quickly, and second, the slope of the drift changes, even becoming negative. In the magnetized case, even if the magnetic field is weak, we have an extra coupling of the interior with the atmosphere due to the magnetic field lines leaving the star's surface. This causes an additional very small transfer of mass and momentum from the atmosphere to the neutron star, which increases the drift in the evolution (see Fig. 5).

The convergence tests show that the order of convergence is 2.13 and 1.56 for model MNS0 and MNS3, respectively. This global order of convergence is consistent with the second-order accuracy of our numerical TVD scheme, which reduces to first order at local extrema such as the center of the star and its surface.

We also compute the Fourier transform of the central density evolution to obtain the mode frequencies of the neutron star pulsations (lower right panel of Fig. 5). We find the fundamental mode frequency at about $f=2.7 \mathrm{kHz}$, and subsequent harmonics at 4.6, 6.4, 8.2, 10.0, 11.8, and $13.8 \mathrm{kHz}$, respectively. Since the energy of the magnetic field is small compared with the potential energy of the star, the influence of the magnetic field on the mode frequency is small. We find no frequency difference between the neutron star models within the frequency resolution $(\sim 0.5 \mathrm{kHz})$. We further observe that the quality of the spectrum deteriorates at higher frequencies for models with stronger magnetic fields. We suspect that this degradation is an artifact due to the stronger coupling of the interior with the atmosphere in the magnetized case.

The second part of the test consists of the evolution of the same neutron star equilibrium models in a dynamic spacetime. For reasons of computational efficiency, the CFC equations are computed only every 100th time step, the metric being interpolated in-between as described by Dimmelmeier et al. (2002a). The results (Fig. 6) are qualitatively the same as those of the Cowling case discussed before. The dynamic spacetime causes larger perturbations in the central density evolution, which now also exhibits a larger drift with time $(<10 \%$ for the $160 \times$ 20 models). Similar drifts were already observed in fully coupled simulations of non-magnetized (Font et al. 2002) and magnetized models (Giacomazzo \& Rezzolla 2007). In both models, MNS0 and MNS3, the drift reduces with increasing resolution, and the order of convergence is 3.1 and 2.5 respectively. The convergence order is higher than expected (second order). We suspect that this is because the $80 \times 10$ zone model is poorly resolved, i.e. the accuracy tends to grow faster than the order of convergence when doubling the resolution. Regarding the comparison between magnetized and non-magnetized models (lower left panel of Fig. 6), we observe larger drifts in the magnetized case due to the stronger coupling with the atmosphere.

The Fourier transform of the central density for the $160 \times$ 20 models with dynamic spacetime evolution (lower right panel of Fig. 6) gives a fundamental frequency of $f=1.4 \mathrm{kHz}$, and higher harmonics at 4.0, 6.0, 7.8, 9.8, 11.6 and $13.7 \mathrm{kHz}$, respectively. We find no dependence on the amount of magnetization within the frequency resolution. A similar result was obtained in the simulations of Montero et al. (2007) regarding pulsating and magnetized thick accretion tori around Schwarzschild and Kerr black holes. This is unsurprising since the normal modes of a star are basically sound waves propagating in the radial direction, and the speed of sound is hardly altered by the magnetization of the investigated models. However, in the magnetized case, new modes can appear due to the richer eigenvalue structure of the GRMHD equations. In particular, it is important to note that Alfvén modes can be excited in the star. For the magnetic field strengths present in our models, these mode frequencies lie below $100 \mathrm{~Hz}$, i.e. much longer simulations are required to be able to see them in the spectrum. A deeper study of the Alfvén modes performed with our numerical code can be found in Cerdá-Durán et al. (2008).

If we compare the frequencies with those in the Cowling approximation, we observe that the Cowling approximation tends to overestimate the frequency of the modes (by almost a factor 2 for the fundamental mode). The higher the order of the harmonics, the smaller is the overestimation, a trend that was observed before in numerical simulations of purely hydrodynamic models (Font et al. 2002). The reason for this behavior is that perturbations on time scales smaller than the typical time scale of variations in the gravitational field (which is roughly $t_{\mathrm{dyn}}$ ) behave similarly as in a fixed spacetime. The frequency corresponding to the dynamic time scale is $f_{\text {dyn }}=10.4 \mathrm{kHz}$. Therefore, modes of frequency higher than $f_{\text {dyn }}$ will be unaffected if the computation is carried out in the Cowling approximation. This agrees with our mode computations.

\subsection{Core collapse}

The final test of our numerical code concerns simulations of magneto-rotational core-collapse. We note that these simulations are not intended to be of astrophysical relevance, since the treatment of neutrinos in the code is still too poor for a study of the 

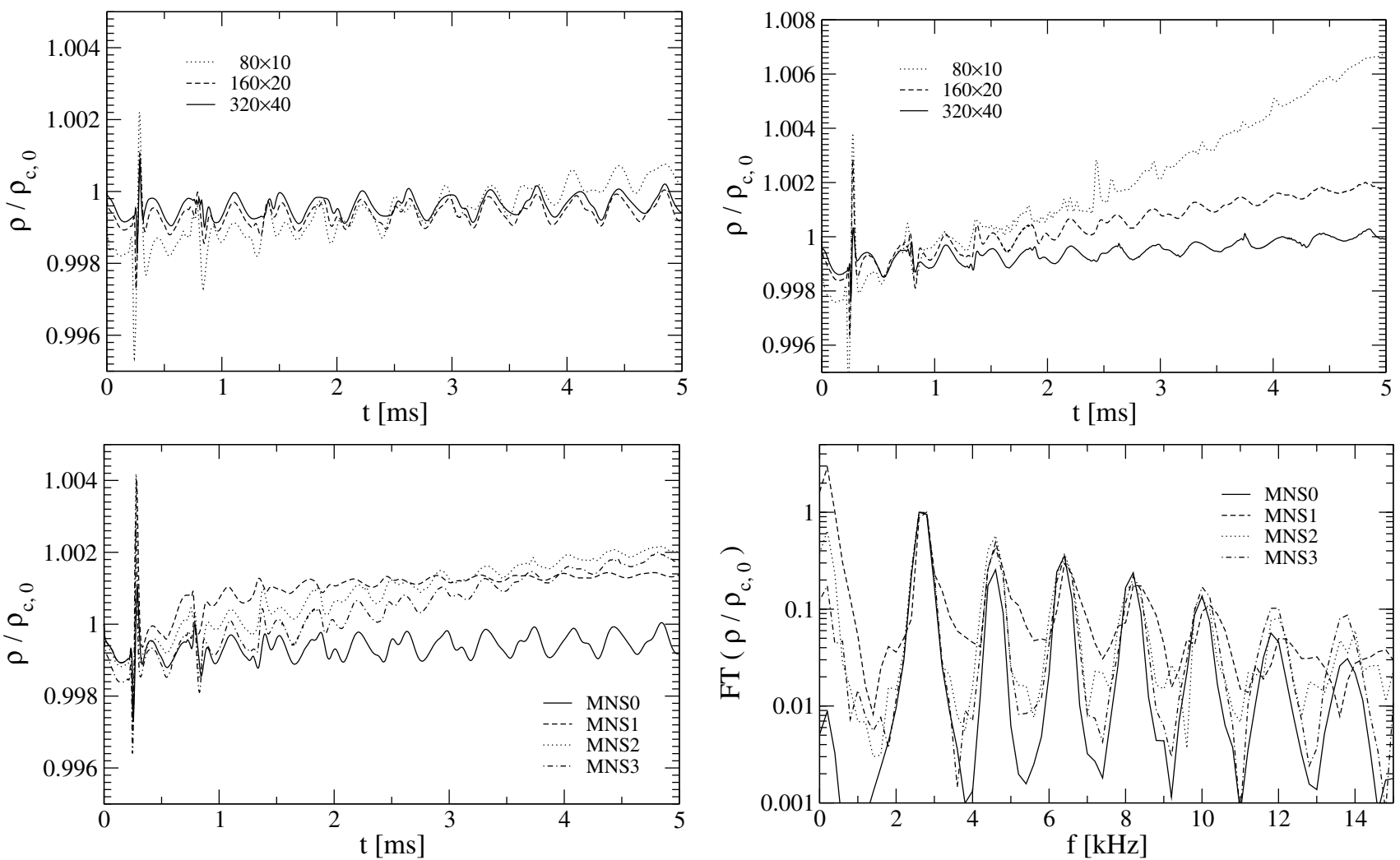

Fig. 5. Evolution of equilibrium configurations of neutron stars in the Cowling approximation (fixed spacetime). The upper panels show the central density normalized to its initial value for the non-magnetized neutron star MNS0 (left) and the magnetized model MNS3 (right). The results are displayed for three different grid resolutions $\left(n_{r} \times n_{\theta}\right)$ : $80 \times 10$ (dotted), $160 \times 20$ (dashed), and $320 \times 40$ (solid). The lower left panel shows the evolution of $\rho / \rho_{\mathrm{c}, 0}$ for all computed models for a grid resolution of $160 \times 20$ : MNS0 (solid), MNS1 (dashed), MNS2 (dotted) and MNS3 (dash-dotted). The lower right panel gives the corresponding Fourier transforms.
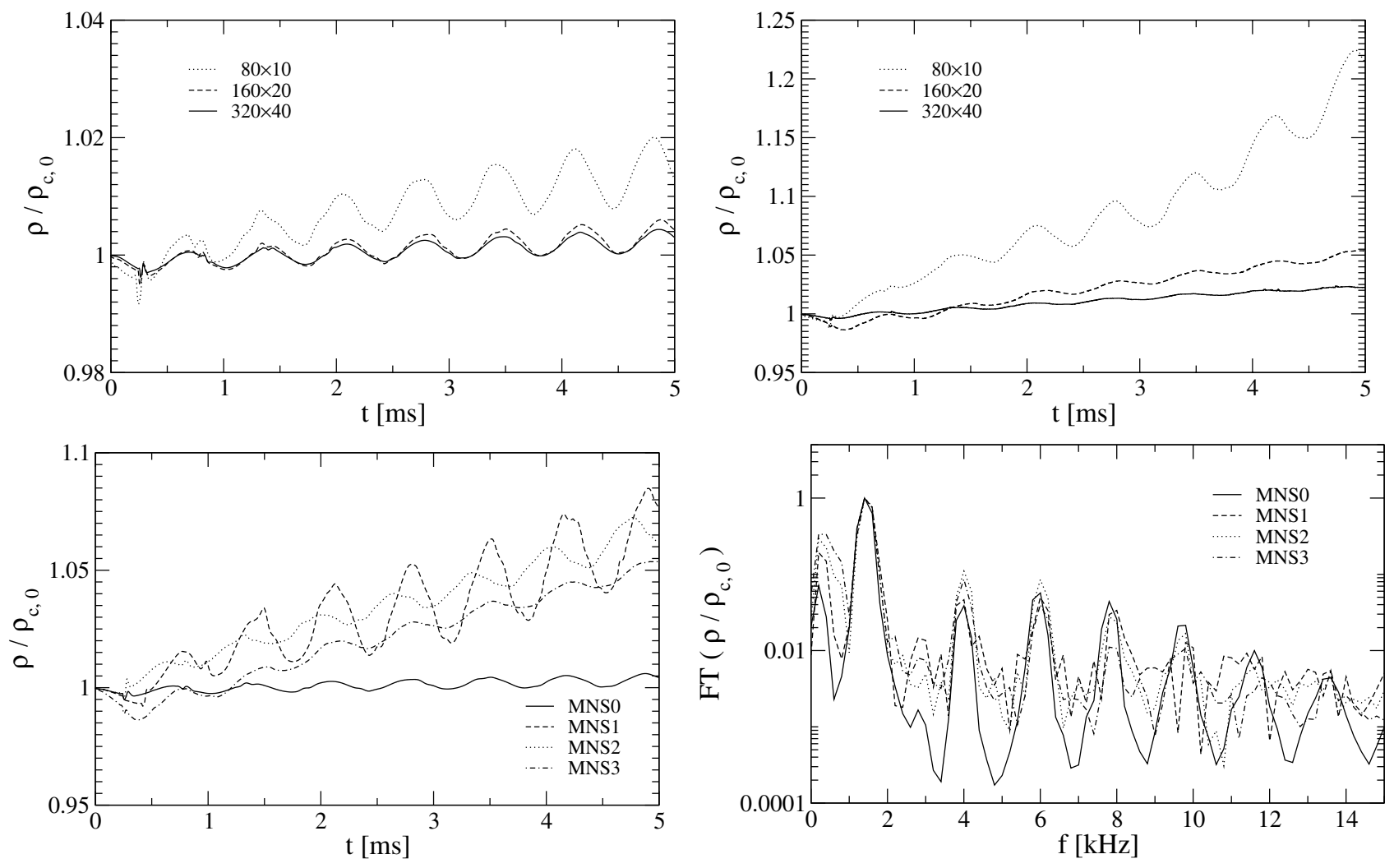

Fig. 6. Same as Fig. 5, but for a dynamic spacetime. 

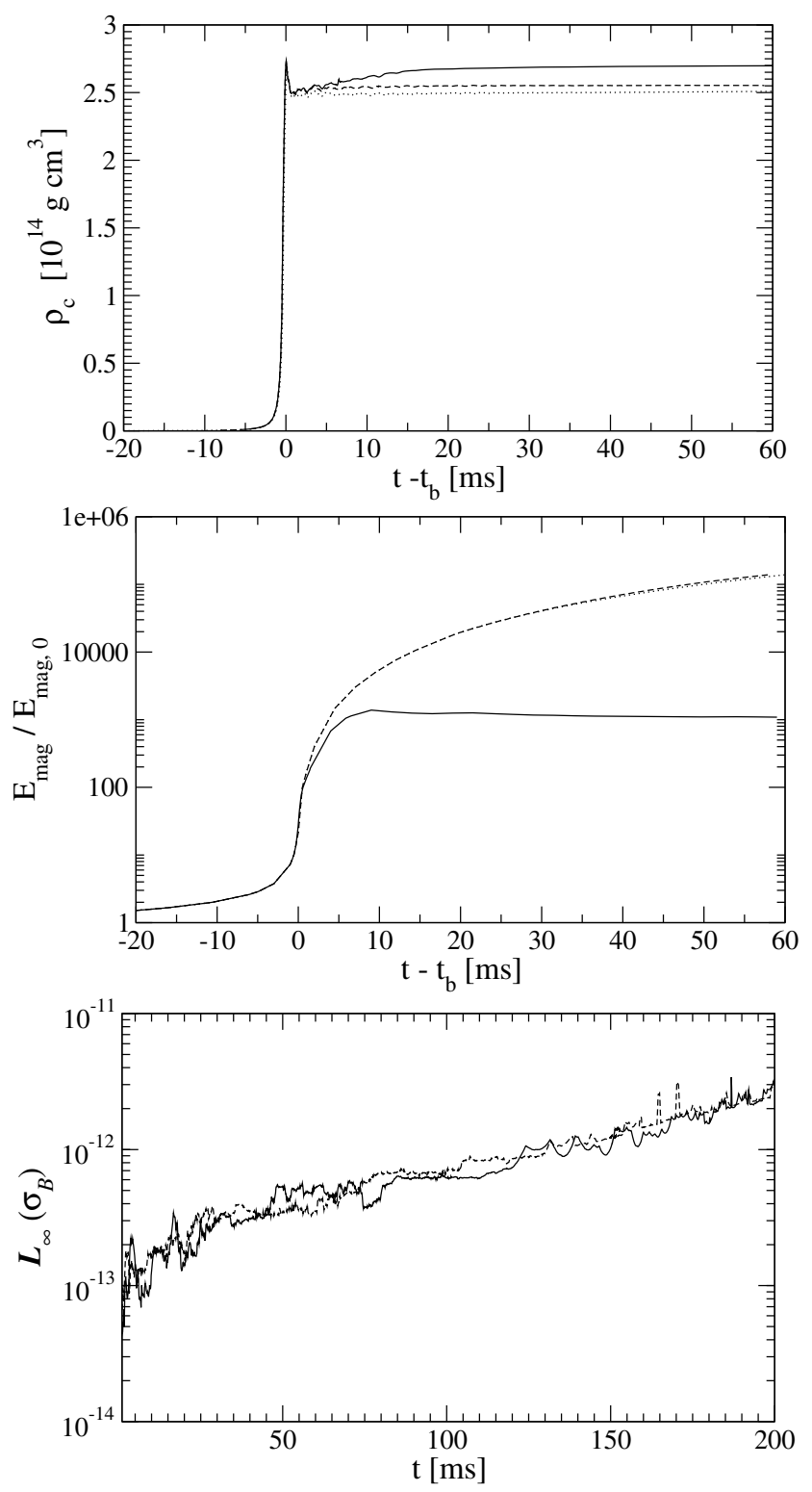

Fig. 7. Evolution of the central density $\rho_{\mathrm{c}}$ (upper panel), of the amplification of the magnetic energy $E_{\mathrm{mag}} / E_{\mathrm{mag}, 0}$ (middle panel) and the $L_{\infty}$ norm of $\sigma_{\mathrm{B}}$ (lower panel) for model s20A1B5-D3M12 (solid), and s20A1B5-D3M10 (dashed), respectively. The results obtained with the passive field approximation (model s20A1B5-D3M0 of Cerdá-Durán et al. 2007) are shown with a dotted line (almost overlapped to the dashed line in the middle panel).

supernova explosion mechanism. Nevertheless, the tests allow us to validate the code in a fully dynamic context including strong magnetic fields, realistic stellar progenitors, and a microphysical EOS. To the best of our knowledge such demanding simulations have not yet been performed, which highlights the unique potential of our new numerical code for the study of relativistic stellar core collapse.

As an initial model, we employ the inner part of the iron core of the solar-metallicity $20 M_{\odot}$ progenitor model of Woosley et al. (2002). To this spherically symmetric and non-magnetized model, we add a rotation profile and a poloidal magnetic field. The rotation law for the specific angular momentum is given by $j=A^{2}\left(\Omega_{\mathrm{c}}-\Omega\right)$, where $A=5 \times 10^{4} \mathrm{~km}$ and $\Omega$ is the angular velocity, which has a value $\Omega_{\mathrm{c}}=4.035 \mathrm{~s}^{-1}$ at center.
The magnetic field is generated by a circular current loop of radius $400 \mathrm{~km}$. This corresponds to model s20A1B5-D3 in Cerdá-Durán et al. (2007) where a more detailed description can be found. We perform simulations for two different initial magnetic field strengths, namely for the weakly magnetized model s20A1B5-D3M10 with a central magnetic field of $|B|_{\mathrm{c}}=10^{10} \sqrt{4 \pi}$ Gauss, and for the strongly magnetized model s20A1B5-D3M12 with $|B|_{\mathrm{c}}=10^{12} \sqrt{4 \pi}$ Gauss. The models are evolved with the tabulated EOS of Shen et al. (1998) and an approximate deleptonization scheme (Liebendörfer 2005) as described by Dimmelmeier et al. (2007), and Cerdá-Durán et al. (2007). We compare the evolutions of these two models with that of the corresponding model s20A1B5-D3M0 of Cerdá-Durán et al. (2007), which was evolved with the passive field approximation. Since the effect of the magnetic field on the collapse dynamics is neglected for model s20A1B5-D3M0, its evolution should be similar to that of our weakly magnetized model s20A1B5-D3M10. The comparison with the passive field model also allows us to identify genuine MHD effects.

Figure 7 shows the evolution of the central density (left panel) and the amplification of the magnetic energy (right panel) for all three models. The latter quantity is computed from

$E_{\mathrm{mag}}=\frac{1}{2} \int \mathrm{d}^{3} \boldsymbol{x} \sqrt{\gamma} W b^{2}$.

Additionally the lower panel of Fig. 7 shows the $L_{\infty}$ norm of $\sigma_{\mathrm{B}}$ defined as the ratio of the total magnetic flux at the surface of each numerical cell to the average magnetic flux on the surface. This dimensionless quantity measures the quality of the numerical preservation of the divergence of the magnetic field along the evolution. The final value is consistent with the round-off error in the evolution, which can be computed as (double precision accuracy) $\times \sqrt{(\text { number of iterations })}=$ $10^{-15} \times \sqrt{5 \times 10^{6}}=2.3 \times 10^{-12}$, if one considers a binomial distribution of errors. As the collapse proceeds both the density and the magnetic energy grow very similarly in all three models, because even in the highly magnetized progenitor model s20A1B5D3M12 the strength of the magnetic field is insufficient to affect the collapse dynamics. The ratio of magnetic energy to gravitational binding energy (see Cerdá-Durán et al. 2007) does not exceed a value of $10^{-7}\left(10^{-3}\right)$ during the collapse in model s20A1B5-D3M10 (s20A1B5-D3M12), which justifies the use of the passive field approximation in the weak magnetic field limit. After core bounce, the low magnetized model s20A1B5-D3M10 continues to behave similarly to model s20A1B5-D3M0, since the magnetic field remains weak. The central density is slightly higher than in model s20A1B5-D3M0, but the magnetic field is far from saturation and is still growing linearly with time at the end of the simulation.

On the other hand, the highly magnetized model s20A1B5D3M12 clearly shows a saturation of the magnetic field energy shortly after core bounce. At this time the ratio of magnetic energy to gravitational binding energy is $7 \%$, a value that is never exceeded during the evolution. Its central density continues to grow beyond bounce, and the model eventually approaches an equilibrium configuration with a central density about $10 \%$ larger than in the passive field case. The behavior of the central density can be understood by examining the angular velocity profiles in Fig. 8. At the time of bounce, the angular velocity profile is very similar for all models, since the magnetic field is still unimportant for the dynamics: the innermost $10 \mathrm{~km}$ of the core rotate rigidly, while further out $\Omega$ follows a power law with an exponent $\sim-1.2$. This profile remains unaltered 

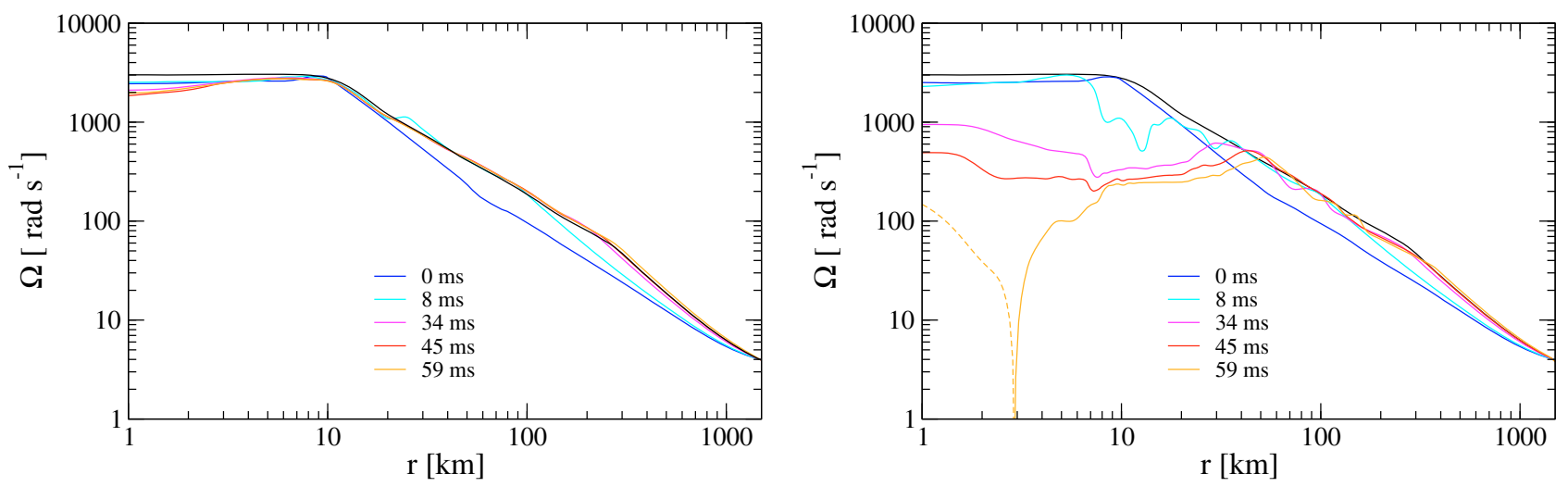

Fig. 8. Radial profiles of the angular velocity $\Omega$ at the equator for model s20A1B5-D3M10 (left panel) and s20A1B5-D3M12 (right panel) at different times after bounce: $t-t_{\mathrm{b}}=0,8,34,45$ and $59 \mathrm{~ms}$. The time-independent rotation profile of the passive field model s20A1B5-D3M0 is shown by the black line in both panels. The yellow dashed line in the right panel indicates a change of sign of the angular velocity.

during the subsequent evolution of the passive field model. In the magnetized models, however, the central region spins down, and the central density rises, the effect being more prominent in the stronger magnetized model s20A1B5-D3M12. The right panel of Fig. 8 shows that the angular velocity begins to decrease for $10 \mathrm{~km} \leq r \leq 30 \mathrm{~km}$ shortly after bounce. In this region, the magnetic field is strongest since differential rotation winds up the magnetic field more efficiently (Cerdá-Durán et al. 2007). On a time scale of about $50 \mathrm{~ms}$, the angular velocity decreases by about a factor 10 , and the innermost few kilometers of the core even acquire retrograde rotation. The reason for this effect is the increasing magnetic tension in the wound-up magnetic field lines. The characteristic time scale in which this magnetic tension acts on the fluid is related to the Alfvén crossing time scale of the innermost region $\tau_{\mathrm{A}} \sim 50 \mathrm{~ms}$, which coincides with the time it takes for the retrograde rotation to appear. This effect was already observed in Newtonian simulations by Müller \& Hillebrandt (1979), and Obergaulinger et al. (2006a). For model s20A1B5-D3M10, the spin-down occurs more slowly, and saturates about $50 \mathrm{~ms}$ after bounce.

To demonstrate the spin-down more clearly, we plot, in Fig. 9, the evolution of the central angular velocity for all three models. In the passive field approximation (black line), $\Omega$ oscillates after bounce in accordance with the oscillations of the core, and approaches a constant value at the end of the simulation. As the magnetic field increases in the progenitor, the spin-down of the core occurs more rapidly. This may be understood by means of the magneto-rotational instability (MRI hereafter). The MRI is a shear instability that can appear when both magnetic fields and differential rotation are present (Balbus \& Hawley 1991), and it gives rise to transport of angular momentum. A necessary condition for the occurrence of the MRI is $\varpi \partial_{\varpi} \Omega^{2}<0$, where $\varpi=r \sin \theta$. In unstable regions, the MRI grows exponentially for all length scales larger than a critical length-scale $\lambda_{\text {crit }} \sim 2 \pi c_{\mathrm{A}} / \Omega$, where $c_{\mathrm{A}}$ is the Alfvén speed. The fastestgrowing MRI mode develops on length-scales near $\lambda_{\text {crit }}$ on a typical time-scale of $\tau_{\text {MRI }}=4 \pi\left[\varpi \partial_{\varpi} \Omega\right]^{-1}$. Therefore, in order to numerically capture the MRI, one has to resolve lengthscales of about $\lambda_{\text {crit }}$. Once the MRI grows, it develops channel flows (Hawley \& Balbus 1992), which are unstable to nonaxisymmetric instabilities (Goodman \& Xu 1994) and eventually become turbulent in three-dimensional simulations (Hawley et al. 1996).

In our simulations, the region with $r>10 \mathrm{~km}$ is unstable to the MRI due to its negative angular velocity gradient. The

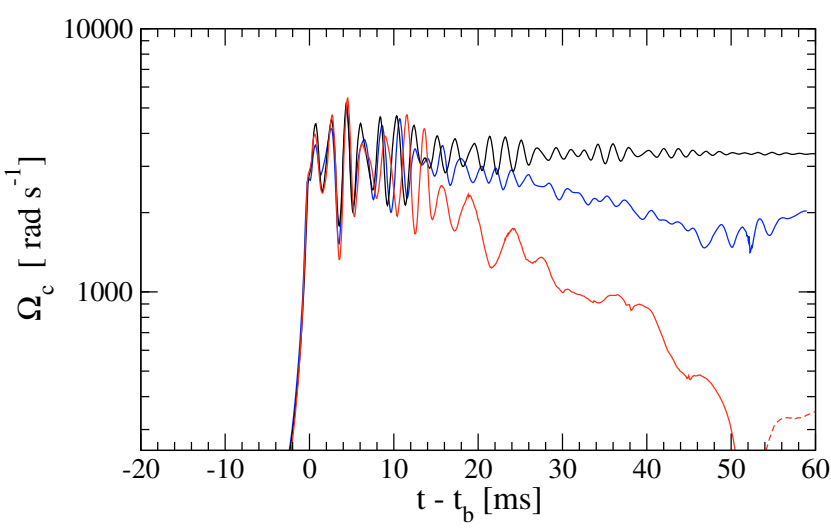

Fig. 9. Evolution of the central angular velocity $\Omega_{\mathrm{c}}$ for model s20A1B5D3M12 (red), s20A1B5-D3M10 (blue), and model s20A1B5-D3M0 (black), respectively. The red dashed line (model s20A1B5-D3M12) indicates a change of sign of the angular velocity.

growth time of the fastest-growing mode is in the range $1 \mathrm{~ms}$ to $10 \mathrm{~ms}$ for the region behind the shock wave, and about $1 \mathrm{~s}$ or even larger further outside. Since the time scale is independent of the initial magnetic field strength, these values are similar for both magnetizations (s20A1B5-D3M10 and s20A1B5-D3M12), and for the passive field case (s20A1B5-D3M0). However, the critical length scale depends on the strength of the magnetic field.

For model s20A1B5-D3M12, the critical length scale at bounce is between $\lambda_{\text {crit }} \sim 1 \mathrm{~km}$ and $5 \mathrm{~km}$ inside the unstable region $(10 \mathrm{~km} \leq r \leq 30 \mathrm{~km})$. This region is covered with $60 \mathrm{ra}-$ dial and 30 angular zones, which corresponds to a resolution $(\Delta r, r \Delta \theta)$ of $125 \mathrm{~m} \times 500 \mathrm{~m}$ at $r=10 \mathrm{~km}$, and $900 \mathrm{~m} \times 1500 \mathrm{~m}$ at $r=30 \mathrm{~km}$. This resolution is marginally sufficient to resolve the length scale of the fastest-growing mode of the MRI at bounce (5-10 radial zones, and 2-3 angular zones). The strong redistribution of the angular momentum observed for model s20A1B5-D3M12 might therefore be caused by the MRI. In turn, the saturation of the magnetic field is a direct consequence of this redistribution of the angular momentum. Without differential rotation, the poloidal magnetic field cannot be wound up into a toroidal magnetic field. The typical spin-down time scale $\tau_{\text {spin-down }}$ can be measured from Fig. 9 by fitting an exponential to the declining part of the curve. For model s20A1B5-D3M12, 


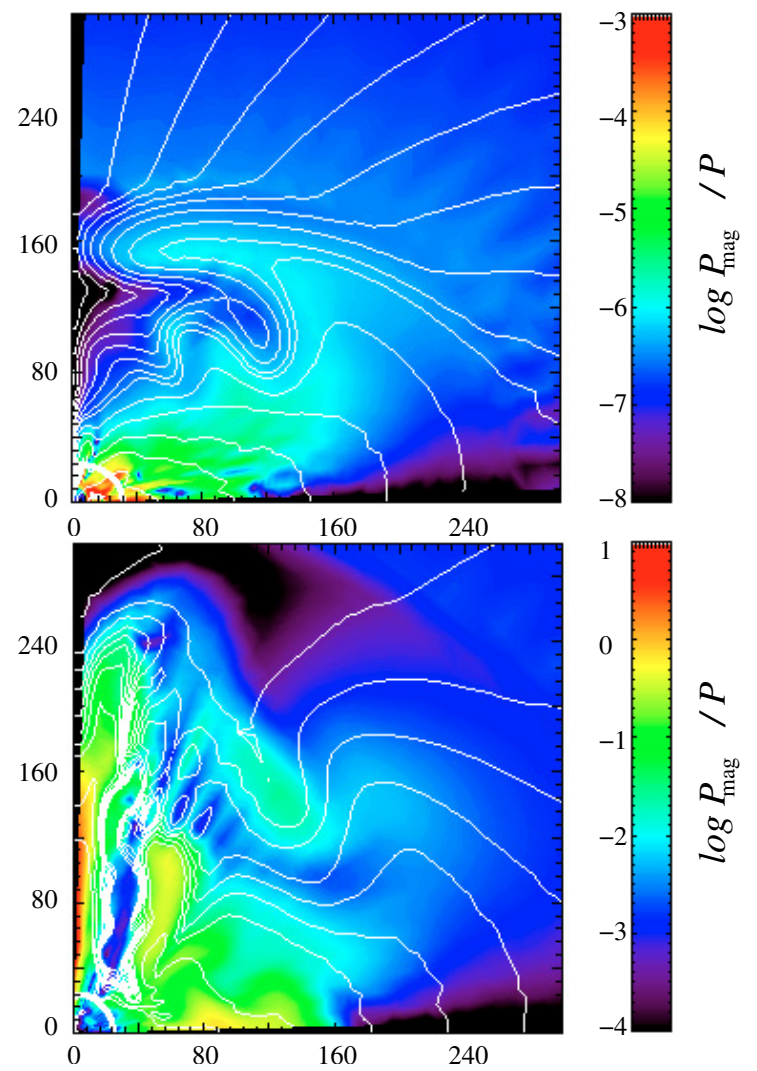

Fig. 10. Magnetic field topology at the end of the simulation, $51 \mathrm{~ms}$ after bounce, for model s20A1B5-D3M10 (upper panel) and s20A1B5D3M12 (bottom panel), respectively. The ratio of magnetic to thermal pressure $P_{\mathrm{mag}} / P$ is shown color-coded. Thin, white lines are poloidal magnetic field lines, and the thick, white line marks the neutrino-sphere. The axis labels are in units of $\mathrm{km}$.

one obtains $\tau_{\text {spin-down }}=22.5 \mathrm{~ms}$, which corresponds roughly to the time scale of the MRI.

On the other hand, for model s20A1B5-D3M10 the critical length-scale at bounce is about a factor of 100 shorter, i.e. between $\lambda_{\text {crit }} \sim 10 \mathrm{~m}$ and $50 \mathrm{~m}$, and thus the fastest-growing mode of the MRI cannot be resolved with our grid resolution. Only modes with slower growth rates can be resolved on the grid. Accordingly, the spin-down for this model occurs on a longer time scale of $\tau_{\text {spin-down }}=62.9 \mathrm{~ms}$. At about $50 \mathrm{~ms}$ after bounce, the innermost $10 \mathrm{~km}$ of the core develops a positive angular velocity gradient (see Fig. 8), and hence becomes stable to the MRI. The central core is no longer able to lose angular momentum, and its spin down stops. We suspect that the appearance of this positive gradient is due to the poorly resolved MRI, which turns out to be more efficient in the inner region, where the resolution is higher, instead of where the shear is larger. The magnetic field continues to grow at similar rates until the end of the simulation due to the further winding-up of poloidal magnetic field lines, and because angular momentum transport is insufficient to affect the rotation profile outside the innermost $10 \mathrm{~km}$ significantly.

Figure 10 displays the magnetic field topology for models s20A1B5-D3M10 and s20A1B5-D3M12 at the end of the simulation. The low magnetized model s20A1B5-D3M10 (top panel) has a similar field structure as model s20A1B5-D3M0 of Cerdá-Durán et al. (2007), since the passive field approximation holds very well for weakly magnetized progenitors (apart from its inability to capture the MRI). The prompt convection ${ }^{2}$ developing after bounce twists the magnetic field outside the neutrinosphere, which is assumed to be located at $\rho_{v}=2 \times 10^{12} \mathrm{~g} \mathrm{~cm}^{-3}$, at about $30 \mathrm{~km}$. In model s20A1B5-D3M12, the magnetic field grows to values close to equipartition, and a distinctive, strongly magnetized outflow propagates along the axis behind the shock front. Between $10 \mathrm{~km} \lesssim r \lesssim 30 \mathrm{~km}$, where the MRI is predominantly growing, axisymmetric channel flows form, which are morphologically similar to the flows found in the simulations of Hawley \& Balbus (1992). We analyze this issue in more detail in Fig. 11, where the development of the channel flows is shown. Their length scale increases as the magnetic field becomes stronger, and since we assume axisymmetry they are stable, i.e. they do not cause any turbulence.

Another important difference between models s20A1B5D3M10 and s20A1B5-D3M12 is the location of the shock. At $\sim 50 \mathrm{~ms}$ after core bounce, the shock is located about $50 \mathrm{~km}$ further out in the strongly magnetized model s20A1B5-D3M12 than in the weakly magnetized model s20A1B5-D3M10. This is most likely a consequence of the transport of angular momentum by the MRI which pushes the shock front to a larger radial distance, although our current grid resolution is probably too poor in the shock region to confirm this interpretation conclusively. Understanding this effect and, in particular, its implications for the explosion mechanism, requires a separate study, which will be published elsewhere.

Since no other simulations yet have been published that are capable of treating a similar combination of general relativity and microphysics, it is impossible to compare directly with other work. Nevertheless, we find qualitative agreement with related simulations of magneto-rotational core collapse (Obergaulinger et al. 2006a,b; Shibata et al. 2006; Burrows et al. 2007). In particular, our simulations share the following aspects with these investigations: (i) redistribution and transport of angular momentum radially outwards due to the MRI, resulting in the spin down of the central region of the core; (ii) increase of the central density after core bounce due to angular momentum losses; and (iii) appearance of a weakly relativistic but highly magnetized outflow along the axis. This agreement strengthens our confidence in the suitability of our new numerical code for the systematic investigation of magneto-rotational core collapse, which we shall report elsewhere.

\section{Conclusions}

We have presented a new numerical code that solves the GRMHD equations coupled to the Einstein equations for the evolution of a dynamic spacetime. Hence, it extends the small list of available codes that are capable of modeling these challenging physics. The main objective of the new code is the study of astrophysical scenarios in which both strong magnetic fields and strong gravitational fields are present, such as the magnetorotational collapse of stellar cores, the collapsar model of GRBs, and the evolution of neutron stars.

Our new numerical code is based on high-resolution shockcapturing schemes to solve the flux-conservative hyperbolic GRMHD equations, and the constraint-transport method to ensure the solenoidal condition of the magnetic field. The Einstein equations are formulated in the CFC approximation, and the

\footnotetext{
2 This transient is produced by an unstable entropy gradient, which is probably an artifact of our poor neutrino treatment. The interested reader is addressed to Cerdá-Durán et al. (2007) for a detailed discussion of this issue.
} 

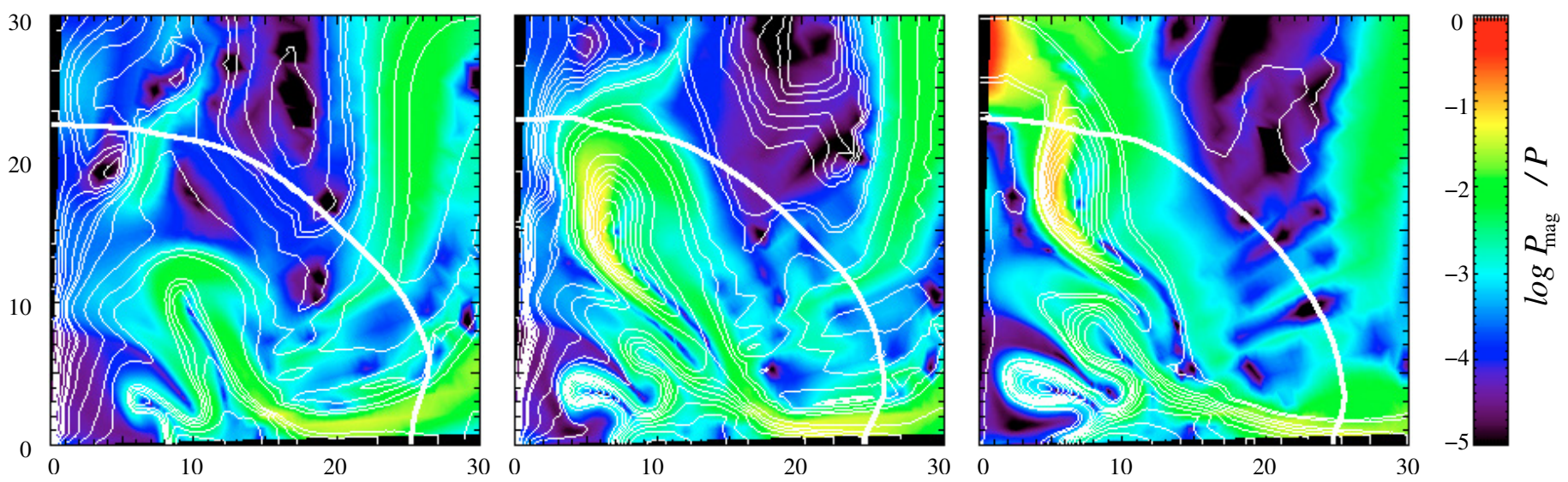

Fig. 11. Details of the magnetic field structure of the core at three different times after bounce: $t-t_{\mathrm{b}}=9 \mathrm{~ms}($ left $), 11.5 \mathrm{~ms}($ middle), and $14 \mathrm{~ms}$ (right), respectively. The ratio of magnetic pressure to thermal pressure $P_{\mathrm{mag}} / P$ is shown color-coded. Thin, white lines are poloidal magnetic field lines, while the thick, white line marks the neutrino-sphere. The axis labels are in units of $\mathrm{km}$.

resulting elliptic equations are solved using a linear Poisson solver. The motivation to use CFC is based on the astrophysical applications envisaged for the code, which do not deviate significantly from spherical symmetry. Furthermore, the code incorporates several equations of state, ranging from simple analytical expressions to tabulated microphysical equations of state.

We have presented a number of stringent tests of our new GRMHD numerical code, which are the main focus of this paper. The test calculations demonstrate the ability of the code to handle properly all aspects appearing in the astrophysical scenarios the code is intended for, namely relativistic shocks, strongly magnetized fluids, and equilibrium configurations of magnetized neutron stars. One of the tests the code has passed successfully is in fact an application, namely the simulation of general relativistic magneto-rotational core collapse using a realistic stellar progenitor model and a microphysical equation of state. We have compared the results obtained by our new code with those of a previous study based on the passive magnetic-field approximation, and find good agreement for initially weakly magnetized progenitors.

Finally, we mention that the new code is also capable of handling the gravitational collapse leading to the formation of a black hole. Results for this specific application will be presented elsewhere. Further extensions of the code that we foresee in the near future include the incorporation of a simplified scheme for neutrino transport (to explore the post-bounce evolution of collapsing magnetized cores more reliably) along with the implementation of resistive MHD.

Acknowledgements. This research has been supported by the Spanish Ministerio de Educación y Ciencia (grant AYA2004-08067-C03-01), and by the Collaborative Research Center on Gravitational Wave Astronomy of the Deutsche Forschungsgesellschaft (DFG SFB/Transregio 7). We would like to thank J. Novak, for the $\mathrm{C}++$ subroutines to import the magnetized equilibrium models from the Lorene code. We also thank the referee, L. Rezzolla, for his useful comments and suggestions.

\section{References}

Anderson, M., Hirschmann, E. W., Lehner, L., et al. 2008, ArXiv e-prints, 801 Anninos, P., Fragile, P. C., \& Salmonson, J. D. 2005, ApJ, 635, 723

Antón, L. 2007, Ph.D. Thesis, Universidad de Valencia, Valencia, Spain, submitted

Antón, L., Zanotti, O., Miralles, J. A., et al. 2006, ApJ, 637, 296

Balbus, S. A., \& Hawley, J. F. 1991, ApJ, 376, 214

Banyuls, F., Font, J. A., Ibáñez, J. M., Martí, J. M., \& Miralles, J. A. 1997, ApJ, 476, 221
Baumgarte, T. W., \& Shapiro, S. L. 1999, Phys. Rev. D, 59, 024007 Bocquet, M., Bonazzola, S., Gourgoulhon, E., \& Novak, J. 1995, A\&A, 301, 757 Burrows, A., Dessart, L., Livne, E., Ott, C. D., \& Murphy, J. 2007, ApJ, 664, 416

Cerdá-Durán, P., \& Font, J. A. 2007, Classical and Quantum Gravity, 24, 155 Cerdá-Durán, P., Faye, G., Dimmelmeier, H., et al. 2005, A\&A, 439, 1033 Cerdá-Durán, P., Font, J. A., \& Dimmelmeier, H. 2007, A\&A, 474, 169 Cerdá-Durán, P., Sotani, H., Stergioulas, N., \& Font, J. A. 2008, in preparation De Villiers, J.-P., \& Hawley, J. F. 2003, ApJ, 589, 458

Del Zanna, L., Bucciantini, N., \& Londrillo, P. 2003, A\&A, 400, 397

Del Zanna, L., Zanotti, O., Bucciantini, N., \& Londrillo, P. 2007, A\&A, 473, 11 Dimmelmeier, H., Font, J. A., \& Müller, E. 2002a, A\&A, 388, 917

Dimmelmeier, H., Font, J. A., \& Müller, E. 2002b, A\&A, 393, 523 Dimmelmeier, H., Ott, C. D., Janka, H.-T., Marek, A., \& Müller, E. 2007, Phys. Rev. Lett., 98, 251101

Dimmelmeier, H., Stergioulas, N., \& Font, J. A. 2006, MNRAS, 368, 1609 Duez, M. D., Marronetti, P., Shapiro, S. L., \& Baumgarte, T. W. 2003, Phys. Rev. D, 67, 024004

Duez, M. D., Liu, Y. T., Shapiro, S. L., \& Stephens, B. C. 2005, Phys. Rev. D, 72, 024028

Evans, C. R., \& Hawley, J. F. 1988, ApJ, 332, 659

Font, J. A. 2003, Living Rev. Relativity, 6, 4, [Online article]: cited on December 11, 2008, http: //www. livingreviews .org/lrr-2003-4

Font, J. A., Goodale, T., Iyer, S., et al. 2002, Phys. Rev. D, 65, 084024 Gammie, C. F., McKinney, J. C., \& Tóth, G. 2003, ApJ, 589, 444 Giacomazzo, B., \& Rezzolla, L. 2007, Classical and Quantum Gravity, 24, 235 Goodman, J., \& Xu, G. 1994, ApJ, 432, 213

Harten, A., Lax, P. D., \& van Leer, B. 1983, SIAM Review, 25, 35 Hawley, J. F., \& Balbus, S. A. 1992, ApJ, 400, 595

Hawley, J. F., Gammie, C. F., \& Balbus, S. A. 1996, ApJ, 464, 690 Ibáñez, J. M. 2006, in A Century of Relativity Physics: ERE 2005, ed. L. Mornas, \& J. Diaz Alonso, Amer. Inst. Phys. Conf. Ser., 841, 100

Isenberg, J. A. 2008, Int. J. Mod. Phys. D, 17, 265

Janka, H.-T., Zwerger, T., \& Mönchmeyer, R. 1993, A\&A, 268, 360

Kelly, P. L., Kirshner, R. P., \& Pahre, M. 2007, ArXiv e-prints, 712

Komissarov, S. S. 1999, MNRAS, 303, 343

Komissarov, S. S. 2005, MNRAS, 359, 801

Komissarov, S. S. 2007, MNRAS, 382, 995

Kössl, D., Müller, E., \& Hillebrandt, W. 1990, A\&A, 229, 378

Kouveliotou, C., Dieters, S., Strohmayer, T., et al. 1998, Nature, 393, 235

Kurganov, A., \& Tadmor, E. 2000, J. Comp. Phys., 160, 214

Leismann, T., Antón, L., Aloy, M. A., et al. 2005, A\&A, 436, 503

Lichnerowicz, A. 1944, J. Math., 23, 3

Liebendörfer, M. 2005, AJ., 633, 1042

Lucas-Serrano, A., Font, J. A., Ibáñez, J. M., \& Martí, J. M. 2004, A\&A, 428, 703

Marek, A., Janka, H.-T., Buras, R., Liebendörfer, M., \& Rampp, M. 2005, A\&A, 443, 201

Martí, J. M. \& Müller, E. 2002, Living Rev. Relativity, 6, 7, [Online article]: cited on December 11, 2008, http: //www . livingreviews . org/lrr-2003-7

Meier, D. L., Epstein, R. I., Arnett, W. D., \& Schramm, D. N. 1976, ApJ, 204, 869

Montero, P. J., Zanotti, O., Font, J. A., \& Rezzolla, L. 2007, MNRAS, 378, 1101

Müller, E., \& Hillebrandt, W. 1979, A\&A, 80, 147 
Nakamura, T., Oohara, K., \& Kojima, Y. 1987, Progr. Theor. Phys. Suppl., 90, 1 Noble, S. C., Gammie, C. F., McKinney, J. C., \& Del Zanna, L. 2006, ApJ, 641, 626

Obergaulinger, M., Aloy, M. A., Dimmelmeier, H., \& Müller, E. 2006a, A\&A, 457, 209

Obergaulinger, M., Aloy, M. A., \& Müller, E. 2006b, A\&A, 450, 1107

Oechslin, R., Janka, H.-T., \& Marek, A. 2007, A\&A, 467, 395

Ott, C. D., Dimmelmeier, H., Marek, A., et al. 2007a, Phys. Rev. Lett., 98, 261101

Ott, C. D., Dimmelmeier, H., Marek, A., et al. 2007b, Class. Quantum Grav., 24, S139

Price, D. J., \& Rosswog, S. 2006, Science, 312, 719

Shen, H., Toki, H., Oyamatsu, K., \& Sumiyoshi, K. 1998, Prog. Theor. Phys., 100,1013

Shibata, M. 1999, Phys. Rev. D, 60, 104052

Shibata, M., \& Font, J. A. 2005, Phys. Rev. D, 72, 047501
Shibata, M. \& Nakamura, T. 1995, Phys. Rev. D, 52, 5428

Shibata, M., \& Sekiguchi, Y. I. 2004, Phys. Rev. D, 69, 084024

Shibata, M., \& Sekiguchi, Y. I. 2005, Phys. Rev. D, 71, 024014

Shibata, M., \& Taniguchi, K. 2006, Phys. Rev. D, 73, 064027

Shibata, M., Liu, Y. T., Shapiro, S. L., \& Stephens, B. C. 2006, Phys. Rev. D, 74, 104026

Shibata, M., Sekiguchi, Y., \& Takahashi, R. 2007, Progr. Theor. Phys., 118, 257 Stergioulas, N., Apostolatos, T. A., \& Font, J. A. 2004, MNRAS, 352, 1089

Tchekhovskoy, A., McKinney, J. C., \& Narayan, R. 2007, MNRAS, 379, 469

Toro, E. F. 1999, Riemann Solvers and Numerical Methods for Fluid Dynamics (Berlin: Springer Verlag)

Tóth, G. 2000, J. Comp. Phys., 161, 605

Wilson, J. R., Mathews, G. J., \& Marronetti, P. 1996, Phys. Rev. D, 54, 1317

Woosley, S. E., Langer, N., \& Weaver, T. A. 1993, ApJ, 411, 823

Woosley, S. E., Heger, A., \& Weaver, T. A. 2002, Rev. Mod. Phys., 74, 1015

Zink, B., Schnetter, E., \& Tiglio, M. 2008, to be published in PRD. 